\title{
Energetic particle transport in compact quasi-axisymmetric stellarators
}

M. H. Redi, H. E. Mynick, M. Suewattana ${ }^{a}$, R. B. White, M. C. Zarnstorff

Plasma Physics Laboratory, Princeton University, P. O. Box 451

Princeton, New Jersey 08540

M. Yu. Isaev, M. I. Mikhailov, A. A. Subbotin

Nuclear Fusion Institute, Russian Research Centre “Kurchatov Institute”, 123182

Moscow, Russia

Abstract

Hamiltonian coordinate, guiding center code calculations of the confinement of suprathermal ions in quasi-axisymmetric stellarator (QAS) designs have been carried out to evaluate the atractiveness of compact configurations which are optimized for ballooning stability. A new stellarator particle following code is used to predict ion loss rates and particle confinement for thermal and neutral beam ions in a small experiment with $\mathrm{R}=145 \mathrm{~cm}, \mathrm{~B}=1-2 \mathrm{~T}$ and for alpha particles in a reactor size device. In contrast to tokamaks, it is found that high edge poloidal flux has limited value in improving ion confinement in QAS, since collisional pitch angle scattering drives ions into ripple wells and stochastic field regions, where they are quickly lost. The necessity for reduced stellarator ripple fields is emphasized. The high neutral beam ion loss predicted for these configurations suggests that more interesting physics could be explored with an experiment of less constrained size and magnetic field geometry.

PACS numbers 52.20.Dq, 52.55.Hc,52.65.Cc,52.65.-y 


\section{INTRODUCTION}

The multi-national, decades-long search for a toroidal magnetic system which will confine stable, high temperature plasma for economical fusion energy has focussed theoretical and experimental investigations primarily on tokamak and stellarator designs. Recent theoretical work by Nuehrenburg ${ }^{1}$ and Garabedian ${ }^{2}$ in addition to experiments at WVII-AS ${ }^{3}$ indicate remarkably good stability and transport properties in a new type of "symmetric stellarator". A stellarator is a toroidal magnetic confinement device with stabilizing field line twist provided largely by external field coils, rather than by plasma current alone ${ }^{4}$. This is expected to allow control of a dangerous energy loss channel, plasma disruptions, driven by plasma current in tokamaks. Stellarators are characterized by good particle confinement as long as the magnetic field magnitude is nearly axially or helically symmetric in magnetic coordinates. These promising characteristics have driven extensive computational exploration of the properties expected in a compact quasiaxisymmetric stellarator $(\mathrm{QAS})^{5}$, of a size similar to the Princeton Beta ExperimentModification (PBX-M) ${ }^{6}$ or the Princeton Large Torus (PLT) ${ }^{7}$ which operated at the Princeton Plasma Physics Laboratory (PPPL) during 1970-1990.

In this paper we investigate the particle confinement behavior of QAS designs which have been scaled in size and magnetic field strength to be similar to the PPPL PBXM experiment. PBX-M achieved high plasma performance, $\beta=7 \%$. $\beta$ is the ratio of the plasma thermal energy to the magnetic field energy and is a figure of merit for the efficiency of magnetic confinement for a specific plasma equilibrium configuration. Such high performance would be desirable to reproduce in a similarly compact stellarator design. If constructed, such an experiment could take advantage of existing heating, 
electrical power and diagnostic capabilities as well as toroidal field coils and a vacuum vessel.

The QAS configurations being investigated are already optimized for ballooning stability $^{8}$ and so are potentially good candidates for interesting plasma physics experiments. In Section II we discuss the magnetic configurations studied and the computational method, which utilizes a new three-dimensional particle following code. Sections III and IV present the transport results for thermal ion and neutral beam ion confinement. Section V summarizes simulations of alpha particle orbits in a reactor sized QAS which is predicted to have good particle confinement at PBX-M size. A summary and conclusion is found in Section VI.

\section{GUIDING CENTER CODE SIMULATIONS OF PARTICLE TRANSPORT IN THREE DIMENSIONAL MAGNETIC CONFIGURATIONS}

Guiding center code simulations which follow the motion of an ensemble of particles in toroidal magnetic coordinates over an energy slowing down time are a wellknown method for the analysis of fast particle loss from tokamak experiments ${ }^{9-15}$. We here make use of a new stellarator version of the Monte Carlo ORBIT $^{16}$ guiding center code, ORBITMN, to assess the loss rate of plasma ions, both thermal and energetic, in promising quasi-axisymmetric stellarator designs. Both ORBIT and ORBITMN calculate particle orbits in Hamiltonian magnetic coordinates with a spline fit to the equilibrium representation of B. ORBIT uses a two-dimensional spline, giving an axisymmetric equilibrium, with capability for adding ripple and MHD modes. ORBITMN uses a harmonic decomposition of the magnetic field obtained from the VMEC $\operatorname{code}{ }^{17}$ and allows 
for up-down asymmetry. It permits analysis of particle confinement in stellarator as well as tokamak geometries.

A toroidal magnetic configuration is described by a set of amplitudes $\left\{\mathrm{B}_{\mathrm{mn}}\right\}$ so that at any radius the magnetic field strength may be written

$$
\mathrm{B}(\mathrm{r})=\sum_{\mathrm{m}, \mathrm{n}} \mathrm{B}_{\mathrm{mn}}(\mathrm{r}) \cos \left[\mathrm{m} \theta+\mathrm{nN}_{\mathrm{p}} \phi\right]
$$

Here $\theta$ is the poloidal coordinate and $\phi$ is the toroidal coordinate, $N_{p}$ is the number of toroidal field periods, and $\mathrm{m}$ and $\mathrm{n}$ are integers describing the poloidal and toroidal harmonic structure of the magnetic field lines. For a tokamak, $N_{p}$ is 1 and if toroidal field ripple is not included (as in this paper) there remain only $n=0$ terms in the characteristic set, $\left\{B_{m n}\right\}$. We obtain the edge toroidal flux $\Phi(a)$ and the $\left\{B_{m n}\right\}$ from a VMEC equilibrium code calculation. VMEC solves the three-dimensional plasma equilibrium equation

$$
\nabla \mathbf{P}=\mathbf{j} \mathbf{x} \mathbf{B},
$$

given the radial plasma pressure and current profiles. In these configurations the pressure profile is taken to be ARIES $^{18}$-like,

$$
\mathrm{P}=\mathrm{P}(0)\left(1-(\Phi / \Phi(\mathrm{a}))^{0.5}\right)^{2},
$$

$\Phi / \Phi$ (a) being the edge normalized toroidal flux, proportional to $r^{2}$. The equilibrium is characterized by high bootstrap current fraction. The field line twist, or q was controlled by the specification of external current transform to be produced by stellarator field coils. Thus VMEC provided a candidate magnetic geometry which was then evaluated for ballooning stability with the TERPSICHORE $\operatorname{code}^{19}$ and subsequently was examined for particle confinement with ORBITMN and $\mathrm{GC}^{20}$. 
Several promising QAS magnetic geometries were found. Parameters for these and related tokamak geometries are shown in Table I and Figs. 1-2. The stellarator and tokamak toroidal configurations were scaled to approximately the size of the PBX-M experimental plasma, $\mathrm{R}=145 \mathrm{~cm}, \mathrm{~B}=1.4 \mathrm{~T}$. Here $\mathrm{R}$ is the position of the magnetic axis. In this way the QAS configurations could be compared and contrasted and some intuitive ideas about important plasma parameters evaluated with realistic computational models. Size and magnetic field strength scaling results are discussed in Sections IV and V.

Table I lists the QAS geometries considered, identified by the number of field periods, the percentage of field line rotational transform (iota $=1 / q$ ) due to external field coils and the average plasma $\beta$, which ranges from 4 to $7 \%$. Each point in a toroidal plasma magnetic equilibrium can be characterized by a parameter $\mathrm{q}=\delta \Phi / \delta \Psi$, the rate of change of the toroidal flux $\Phi$ with poloidal flux $\Psi$; the plasma magnetic shear, $\mathrm{s}=$ $(1 / \mathrm{q}) \mathrm{dq} / \mathrm{dr}$, is the dimensionless radial derivative of this plasma safety factor, q. This is denoted the safety factor because low $q(0)$ and regions of rational $q$ are associated with MHD unstable equilibria. In addition, high q drives large stochastic ripple diffusion losses.

The table includes two-field period stellarators with average $20 \%$ and $40 \%$ external transform, and a four-field period stellarator with $40 \%$ external transform which were found to be ballooning stable. These three stellarators also were quite compact and had very low aspect ratio, $\mathrm{a} / \mathrm{R}=2.1$. A stellarator with three field periods and somewhat higher aspect ratio $(\mathrm{a} / \mathrm{R}=3.5)$ was found to have good ballooning stability but to be kink unstable (QAS3_34). The related three-field period stellarator denoted QAS3_53 was then generated by optimization, which has higher external transform to 
minimize ripple transport, montonically increasing transform to suppress tearing modes and high edge shear to provide kink stability. However this configuration, also designated QAS3C10 by the NCSX team, will be seen to show poor fast ion confinement and was not fully kink stabilized. Some of the stellarator designs were scaled from B=1.4 $\mathrm{T}$ to B $=2.8 \mathrm{~T}$ and to $1 \mathrm{~T}$, to evaluate the effect of increased and decreased magnetic field and were scaled to reactor size $(\mathrm{R}=453 \mathrm{~cm}, \mathrm{~B}=7.9 \mathrm{~T})$ for alpha particle transport calculations.

The Table also lists parameters of three related tokamaks for which comparative simulations were done. TOKQAS2_20 is an "equivalent tokamak" similar to QAS2_20, but without contribution to iota from external field coils. This "equivalent tokamak" has the same $\mathrm{n}=0$ components of the set $\left\{\mathrm{B}_{\mathrm{mn}}\right\}$, which define the boundary, as well as the same total plasma current, toroidal flux, and pressure profile as QAS2_20. The only difference is the radial or flux coordinate dependence of the set $\left\{B_{m n}\right\}$, due to the difference in equilibria. TOKQAS2_20q is also an "equivalent tokamak", but one which is constrained to have the same q profile as QAS2_20 and therefore has lower plasma q than TOKQAS2_20. It has the same flux surface plot as TOKQAS2_20. The TOK0 geometry is obtained from an analytic equilibrium for a high aspect ratio, 6\% beta tokamak, with R and B as in the other QAS configurations.

Figure 1 shows the flux surfaces for the toroidal magnetic configurations and Figure 2 shows the q profiles. Flux surfaces and q profiles for QAS3_53 are similar to those of QAS3_34 and are not shown. 


\section{SIMULATIONS OF THERMAL ION TRANSPORT}

To study thermal ion transport, the ORBITMN guiding center code simulations followed a monoenergetic ensemble of 1000 to 4,000 particles, at $7 \mathrm{keV}$, in the equilibrium toroidal magnetic geometry of a $\mathrm{T}_{\mathrm{eo}}=3.5 \mathrm{keV}$, deuterium plasma. Good confinement of these Maxwellian tail ions would confirm good neoclassical plasma confinement for a proposed experiment. A particle is declared lost when it reaches the last closed flux surface. The ORBITMN simulations used only the 50 harmonics from the set $\left\{B_{m n}\right\}$ which have the highest peak amplitudes, out of a set of 304 harmonics obtained by VMEC. The guiding center code simulation of particle orbits neglects fast gyro-motion, but includes collisional effects. Slowing down processes were neglected but pitch angle scattering $v_{\mathrm{pa}}=v_{\perp} / 2$ was included for $\mathrm{N}_{\mathrm{eo}}=3 \times 10^{13} / \mathrm{cm}^{3}$, Zeff $=2.5$. The collision frequency was assumed to have a radial dependence $v_{\perp}={v_{\perp}}_{\perp}(1-\Phi / \Phi(a))$, where $\Phi$ is the radial position in toroidal flux coordinates with $v_{\perp}^{0}=324 / \mathrm{sec}$.

From particle orbit motions, we calculate the diffusion coefficients of the ions and the loss fractions. Figure 3 shows the initial ion density profiles for thermal ions which were launched at $r=a / 2$, with random poloidal, toroidal and pitch angle distributions. Figs. 4-6 show simulation results for six of the toroidal geometries studied. Figure 4 shows the final ion density profiles for 1000 particles after 400 toroidal transits (the time for one toroidal transit of an ion at the magnetic axis with velocity parallel to the magnetic field line). Figure 5 shows the thermal ion loss fraction as a function of elapsed time. In Table II are shown the results for the loss fractions and diffusion coefficients obtained from the 1000 particle, 400 transit time simulations and for improved accuracy, from simulations of 4000 particles for 40 transit times. 
In order to evaluate the diffusion coefficient, $\mathrm{D} \sim \Delta \mathrm{r}^{2} / 2 \mathrm{t}$, we define $\mathrm{X}_{\mathrm{k}}=\left[\Phi_{\mathrm{k}} / \Phi(\mathrm{a})\right]$ with $\Phi_{\mathrm{k}}$ being the radial position of the kth ion in toroidal flux coordinates and $\Phi(\mathrm{a})$ the edge toroidal flux. Also $\mathrm{X}_{\mathrm{k}_{\mathrm{o}}}=\left[\Phi_{\mathrm{k}_{\mathrm{o}}} / \Phi(\mathrm{a})\right]$, where $\Phi_{\mathrm{k}_{\mathrm{o}}}$ is the initial position of the kth ion in toroidal flux coordinates. Fig. 6 shows the evolution of

$$
f(t)=1 /\left(2 N_{k}\right) \sum_{k}\left(X_{k}^{0.5}-X_{k_{0}}^{0.5}\right)^{2} a^{2}
$$

$\mathrm{N}_{\mathrm{k}}$ is the number of particles in the ensemble and $\mathrm{a}=$ midplane average radius at toroidal angle $\phi=0$. The diffusion coefficients $\mathrm{D}$ in the Table are obtained from $\mathrm{D}=\delta \mathrm{f} / \delta \mathrm{t}$ $(<a>/ a)^{2}$, where $\delta f / \delta t$ has been scaled by the square of the ratio of the stellarator-averaged minor radius to the midplane stellarator average radius at $\phi=0$.

For accurate determinations of $\mathrm{D}$ it is important that the simulation not lose a large ion fraction from the last closed flux surface. Figure 5 and Table II show that all the QAS except QAS2_20 have loss fractions greater than 10\% after 400 toroidal transits. Such high loss rates give erroneous estimates for D, as "lost" particles are held fixed at the plasma edge and the high diffusion of the configuration does not continue to be reflected in the accumulating quantity $f(t)$. The diffusion coefficients for most of the stellarator designs were evaluated with 4000 particle, 40 toroidal transit time simulations. These simulation times were held short enough to give total losses less than about $5 \%$. In some cases D was not evaluated where particle transport was intrinsically very high.

These stellarators have complex three-dimensional regions with different stochastic ripple thresholds, unlike the rather well-defined two-dimensional confinement domains of tokamaks, for example see Ref. 12. In contrast to the tokamak case, the behavior of plasma ions in QAS is not a simple diffusion, but a sum of processes for the threshold criteria. Comparing the fractional losses in Table II for 40 transit times and for 
400 transit times, we would expect, since the time increases by 10 , that the loss fraction would increase by $\sim 10^{0.5} \sim 3$, if diffusive loss was dominant. The TOK0 configuration does show this behavior. QAS4_40 is the only one of the stellarator configurations considered which has an overall diffusive behavior. All the other stellarator configurations show greater losses than simple diffusion would predict, in some cases by more than an order of magnitude (viz. QAS2_20, QAS3_34, QAS3_53). This indicates that the ion motion is primarily due to slow stochastic field ripple diffusion, or to slow stellarator ripple trapping loss, whre the ion follows a helical path. This last is very different from the vertical drift motion found for tokamak ripple trapped ion loss.

We find in Table II that the QAS2_20 design has particle transport very similar to that of an equivalent tokamak with no external field coils, TOKQAS2_20, (D 8000 $\mathrm{cm}^{2} / \mathrm{sec}$ ). This equivalent tokamak has lower iota, and higher plasma $\mathrm{q}$ than the QAS. The QAS2_20 and TOKQAS2_20 diffusion coefficients are about twice as high as TOKQAS2_20q, the tokamak of the same plasma size and magnetic field, but which has the same $\mathrm{q}(\mathrm{r})$ profile as QAS2_20.

The Table shows that particle transport in QAS configurations was poorer than in the equivalent tokamak, particularly when q was held fixed. This can be seen for both the two field period and three field period cases. Although QAS2_40 and QAS4_40 have high diffusion coefficients, good particle transport can be achieved with a high fraction of external iota as shown by the low diffusion coefficient of QAS3_53.

For quasi-axisymmetric toroidal magnetic configurations, the canonical momentum of passing ions will be quasi-conserved, so that as for tokamaks we might expect QAS particle confinement to be better for high edge poloidal flux ${ }^{21}$. Table III shows the 
diffusion coefficients along with parameters for each magnetic configuration which neoclassical theory predicts will affect particle transport and the value of D: the edge q, the edge poloidal flux, $\Psi_{\text {edge }}$, in addition to the stellarator-averaged radius $\langle$ a $\rangle$, and the outer midplane radius at $(\theta=0, \phi=0), \mathrm{a}_{0,0}$, and a measure of the stellarator field line ripple, W, discussed in Section IV.

We see that tokamak confinement is indeed improved by high $\Psi_{\text {edge }}$ and by greater distance between the plasma magnetic axis and the last closed flux surface, $a_{0,0}$. This is equivalent to smaller particle losses being correlated to a small ratio of the ion banana width to average minor radius, $\rho / a$. Note the higher loss rates (Table II) for the high aspect ratio tokamak, TOK0, which has smaller $\Psi_{\text {edge }}$, and <a>, than TOKQAS2_20.

The diffusion coefficients found from thermal ion transport simulations are shown in Fig. 7 versus the edge poloidal flux for $\mathrm{B}=1.4$ T. For the equivalent tokamak cases, the particle confinement does improve as the edge poloidal flux increases. For the tokamaks, the decrease in D is not as large as expected from the $\mathrm{q}^{2}$ dependence of analytic models for tokamak neoclassical diffusive transport in the banana regime ${ }^{22}$. This is because the thermal ions are not deep in the banana regime; $v^{*}$ is not sufficiently lower than 1 (Table IV). High values of $q$ and large ion banana widths effectively delocalize particle motion, making the analytic, zero banana width neoclassical model inaccurate.

The overall trend of the QAS particle confinement does not improve with higher edge poloidal flux. The best QAS confinement is found for QAS2_20, QAS3_34, and QAS3_53. But when QAS3_53, is scaled to $1 \mathrm{~T}$ and compared to the equivalent tokamak (same q profile), we find five times greater losses in the QAS than in the tokamak (Table II, $17 \%$ vs 3\%). 
The ORBITMN code has been benchmarked against the Mynick particle transport code, $\mathrm{GC} 3^{20}$, which found $\mathrm{D}=7426 \mathrm{~cm}^{2} / \mathrm{sec}$ for thermal ion transport in QAS2_20. This is in satisfactory agreement with the ORBITMN diffusion coefficient, $\mathrm{D}=8570 \mathrm{~cm}^{2} / \mathrm{sec}$; the ORBITMN diffusion coefficients are estimated to have an accuracy of $\pm 20 \%$.

This Section has surveyed thermal particle transport in a set of QAS designs of PBX-M size, characterized by magnetohydrodynamic (MHD) ballooning mode stability. We wished to see whether a PBX-M size QAS plasma at density and temperature similar to PBX-M experiments would have low enough neoclassical transport that interesting experiments could be carried out over times $\sim 30 \mathrm{msec}$, typical of those experiments. Anomalous transport, which is not yet well understood theoretically, could then be studied. Since a characteristic particle confinement time can be estimated from $D=a^{2} / 4 \tau$, with $\mathrm{a}=20 \mathrm{~cm}$ and $\mathrm{D}=8000 \mathrm{~cm}^{2} / \mathrm{sec}$, the plasma would be confined for a time $\sim 13 \mathrm{msec}$ in QAS2_20. Simulations of QAS3_53 with the magnetic field reduced to $1 \mathrm{~T}$, instead of 1.4 T, show larger particle loss fractions and reduced particle confinement. This design at 1T loses more than 5 times as many plasma ions as does the equivalent low q tokamak, which would confine plasma for a time $\sim 28 \mathrm{msec}$. It will be necessary to further reduce the magnetic field ripple in these QAS designs to obtain performance comparable with that found in PBX-M.

\section{SIMULATIONS OF NEUTRAL BEAM ION TRANSPORT}

In the previous Section we presented simulations of thermal particle transport in several QAS of PBX-M size. In the following two sections we describe the results of 
simulations of neutral beam orbits corresponding to experiments in these QAS configurations with beam ion parameters similar to $\mathrm{PBX}-\mathrm{M}$, with beam ion profiles having low and high edge deposition.

\section{Section IV.A Simulations for Low Edge Beam Deposition}

Initial simulations were carried out with a radial deposition profile as calculated by the TRANSP ${ }^{23}$ analysis code for $40 \mathrm{keV}$ deuterium, neutral beam ions given by the flux surface averaged, total beam ion density profile (Figure 8 , low edge deposition profile) for PBX-M shot 60678 , which reached $7 \% \beta$. This profile was calculated by TRANSP to include all beam line orientations and all beam ion energies, but does not include ions lost to first orbit and charge exchange. The ORBIT simulations modelled only the full energy ions with this TRANSP profile for each beam line. The profile is broader, but with lower edge deposition than the full energy, co-parallel beam deposition profile used for simulations discussed in the next section. Unless otherwise noted, beam ions were launched at the midplane, $\theta=0$, with random toroidal angle $\phi$, with pitch, $\lambda$, determined by the tangency radius $\left(\mathrm{R}_{\tan }\right)$ corresponding to each of the four PBX-M beam lines $(\lambda=$ $\left.\left[\mathrm{R}_{\mathrm{tan}} / \mathrm{R}\right][\mathrm{B}(0) / \mathrm{B}(\mathrm{r})]\right)$.

The collision frequencies were estimated using standard formulations ${ }^{24}$, which depend on the electron density, the electron temperature and the level of plasma impurities. The energy slowing down rate is given by $v_{\varepsilon}=v_{\varepsilon}^{\text {ie }}+v_{\varepsilon}^{\text {i,thermal ions }}+v_{\varepsilon}^{\text {i,impurities. }}$ Here $v_{\varepsilon}^{\text {ie }}=2 v_{\mathrm{s}}{ }^{\text {ie }}-v_{\perp}$ ie $-v_{/ /}{ }^{\text {ie }}$; each term is proportional to $\mathrm{N}_{\mathrm{e}}$. The second and third terms in the energy loss rate, $v_{\varepsilon}^{\mathrm{i}, \text { thermal ions }}+v_{\varepsilon}^{\mathrm{i} \text {,impurities }}$, are each proportional to $\mathrm{N}_{\mathrm{i}} \mathrm{Z}_{\mathrm{i}} / \mu_{\mathrm{i}}$. The pitch angle scattering collision rates include scattering from thermal ions and impurities as 
well as a tiny contribution from electrons: $v_{\perp}=v_{\perp}{ }^{\text {ie }}+v_{\perp}{ }^{i \text {,thermal ions }}+v_{\perp}$ i,impurities . Whereas $v_{\perp}$ ie is proportional to $N_{e}$, the sum $\left\{v_{\perp}{ }^{i, \text { thermal ions }}+v_{\perp}{ }^{\text {i,impurities }}\right\}$ is proportional to $N_{e} Z_{\text {eff }}$.

The background plasma was taken to be primarily deuterium with Zeff set equal to 1.8 , rather than the more typical 2.7 , and with average plasma temperature and densities similar to PBX-M experiments. The volume average plasma parameters are $\mathrm{T}_{\mathrm{e}}=1.3 \mathrm{keV}, \mathrm{N}_{\mathrm{e}}=4 \times 10^{13} / \mathrm{cm}^{3}, \mathrm{~N}_{\mathrm{imp}}=0.1 \times 10^{13} / \mathrm{cm}^{3}, \mathrm{~N}_{\text {thermal ions }}=2.6 \times 10^{13} / \mathrm{cm}^{3}, \mathrm{Z}_{\mathrm{imp}}=9$, $\mathrm{Z}_{\text {eff }}=1.8, \mu_{\mathrm{imp}}=18, \mu_{\text {thermal ions }}=2$. For this case the energy slowing down collision frequency becomes

$$
\begin{aligned}
& v_{\varepsilon}=v_{\varepsilon}^{\text {ie }}+v_{\varepsilon}^{\text {i,thermal ions }}+v_{\varepsilon}^{\text {i,impurities }}=37 / \text { sec } \\
& \text { since } v_{\varepsilon}{ }^{i e}=2 v_{\mathrm{s}}{ }^{i e}-v_{\perp}{ }^{i e}-v_{/ /}{ }^{i e}=2 \times 13.7-0.9-0.4=26 / \mathrm{sec} \\
& \text { and } \quad v_{\varepsilon}^{i, \text { thermal ions }}+v_{\varepsilon}^{\text {i,impurities }}=8.3+2.9=11 / \text { sec. }
\end{aligned}
$$

The perpendicular diffusion rate, related to the pitch angle scattering rate by $v_{p a}=v_{\perp} / 2$, is

$$
\begin{gathered}
v_{\perp}=v_{\perp}{ }^{\text {ie }}+v_{\perp}{ }^{\text {i,thermal ions }}+v_{\perp}{ }^{\text {i,impurities }}=24 / \mathrm{sec} \\
\text { since } \quad v_{\perp}^{\text {ie }}=0.9 / \mathrm{sec} \text { and } v_{\perp}{ }^{\text {i,thermal ions }}+v_{\perp}{ }^{\text {i,impurities }}=23 / \mathrm{sec}
\end{gathered}
$$

Note that the frequencies roughly satisfy the ratio $v_{\perp} / \nu_{\varepsilon}=0.7$. We take $v_{\varepsilon}$ to be constant with radius and give $v_{\perp}$ the radial dependence $v_{\perp}=v_{\perp}^{0}(1-\Phi / \Phi(a))$, making $v_{\perp}(0) \sim 35 /$ sec.

The results of the ORBITMN neutral beam ion simulations are given in Tables VIX. In Table $\mathrm{V}$ are shown simulation loss rates for the QAS2_20 stellarator with the collision frequencies calculated above. Beam ions can be classified as counter-injected or co-injected with respect to the direction of the plasma current. Ion drift causes the banana orbits of counter-injected beam ions to be displaced from the flux surface toward the vessel wall and, consequently, counter injected beams exhibit a fraction of prompt or first orbit losses while co-injected beams do not. In addition, beam ions are classified as 
injected perpendicular or parallel to the plasma magnetic axis. Beam lines which are injected perpendicular produce primarily trapped ions and will have higher loss fractions due to stochastic ripple and first orbit loss (if they are also counter injected).

Table $\mathrm{V}$ shows the loss fractions are minimal for parallel neutral beam ion injection in the direction parallel to the plasma current. For perpendicular injection, trapped particle loss due to scattering into stochastic loss regions or ripple wells is predicted to be high $(70-84 \%)$ and would preclude practical neutral beam ion experiments. Counterinjected beam ions even in the parallel direction, exhibit much scattering into prompt-loss orbits. Simulation results without pitch angle scattering make clear the strong effect of collisional scattering into the stochastic regions and ripple wells. Simulations for the Tokamak Fusion Test Reactor (TFTR) ${ }^{25}$ showed that the addition of collisions to collisionless TF ripple loss doubles the ripple loss fraction in typical supershot plasmas for both neutral beam ions and alpha particles ${ }^{9}$. For a QAS this synergistic effect is even greater because of the multitude of stochastic regions. The addition of collisions increases the loss of energetic ions by a factor of 2 to 50 depending on injection angle. We also see in Table $\mathrm{V}$ that if the magnetic field strength is increased to $2.8 \mathrm{~T}$, energy and particle loss fractions (averaging $40 \%$ and 54\% over all beam lines) are reduced by $1 / 2$ for parallel injection. For comparison the ripple loss of beam ions in TFTR was calculated to be about $10-20 \%{ }^{9}$.

It is often claimed that it is only the ratio $v_{\perp} / v_{\varepsilon}$ which determines the ripple driven loss rate of energetic ions in a tokamak or stellarator. But the length of the slowing down time can also be an important determining factor in these loss rates. In Table VI are shown the ORBITMN simulation results for the same ratio of $\left\langle v_{\perp} / v_{\varepsilon}\right\rangle$ as for Table $V$ but 
with lower average energy slowing down collision frequency, $17 / \mathrm{sec}$. The loss rates increased by more than a factor of two when the slowing down time doubled between the two sets of simulations. To decrease energetic ion losses, the ratio $\left\langle v_{\perp} / v_{\varepsilon}\right\rangle$ and the ion slowing down time should be kept as small as possible. The slowing down time can be reduced by keeping the plasma temperature low and the density high, while the ratio $\left\langle v_{\perp} / V_{\varepsilon}\right\rangle$ can be reduced by keeping $Z_{\text {eff }}$ low.

In Table VII are shown the ORBITMN results for simulations of parallel coinjected neutral beam ions for five QAS and two equivalent tokamaks of PBX-M size. The high loss fraction in TOKQAS2_20 is due to the high value of q, and low edge poloidal flux of that tokamak. We find the lowest beam ion losses for QAS configurations in the QAS2_20 and QAS3_34 designs.

The QAS3_53 design is of special interest as it was optimized from the QAS3_34 configuration for increased edge shear toward improved kink stability. It is however not kink stable at $4 \%$ beta but was considered sufficiently intesting that the NCSX team used this configuration as a group design point in 1999. We examined the thermal ion transport for QAS3_53 in the previous Section and in Table VIII show the simulation results for neutral beam ion loss in a PBX-M device with $\mathrm{B}=1 \mathrm{~T}$. For this case, the simple approximation to the beam ion profile was replaced by a more detailed TRANSP analysis calculation of the deposited beam ion distribution function $f(\lambda, E, r)$, at $40 \mathrm{keV}$, projected to the plasma midplane. This more accurate distribution function gave loss rates within $10 \%$ of those obtained with the simpler beam ion profile. Energetic ion loss rates are four times greater for the QAS3_53 design than for the QAS2_20 configuration, although thermal ion diffusion coefficients are within a factor of two. Although good thermal 
particle confinement was found for both QAS3_53 and QAS2_20, it turned out to be necessary to do both thermal and energetic ion simulation studies when searching for an optimal configuration with good fast ion confinement. The $28 \%$ neutral beam ion loss from QAS3_53 is marginally acceptable for a proposed experiment.

The simulations have not included estimates of charge exchange loss (with characteristic time $\tau_{\mathrm{cx}}$ ) or effects of stellarator ripple fields on electron heating nor electric field effects on neutral beam ion loss. Goldston and Towner $^{26}$ showed in 1981 that collisional ripple transport does not prevent tangential neutral beam injection from being effective in heating, even for highly non-axisymmetric systems such as stellarators, as long as $\mathrm{T}_{\mathrm{e}} / \mathrm{E}_{\mathrm{nb}}$ and $\tau_{\varepsilon} / \tau_{\mathrm{cx}}$ are small.

The neutral beam ion loss fractions are plotted versus edge poloidal flux in Figure 9. In general the goodness of energetic ion transport in 1.4 T QAS stellarators exhibits a pattern similar to that seen for thermal ion transport in Fig. 7. Figure 9 shows the strong dependence on edge poloidal flux of neutral beam ion loss expected for coinjected parallel beams in tokamaks of the same size and field as the QAS configurations. The QAS3_53 design shows only slightly reduced losses as poloidal edge flux is increased. For the QAS configurations, high edge poloidal flux is a necessary but not sufficient condition for good neutral beam ion confinement.

\section{Section IV.B Simulations for High Edge Beam Deposition}

Simulations were also carried out with a radial profile of $40 \mathrm{keV}$ deuterium, neutral beam ions given by the flux surface averaged, beam ion deposition as calculated by the TRANSP $^{23}$ analysis code for the QAS3_53 configuration with co-parallel only injection. 
Figure 8 shows this high edge deposition beam profile. Again, the beam ions were launched at the midplane, $\theta=0$, with random toroidal angle $\phi$, with pitch, $\lambda$, determined by the tangency radius $\left(\mathrm{R}_{\mathrm{inj}}\right)$.

With the higher edge deposition profile, the equivalent tokamak geometry (axisymmetric component of QAS3_53) at $\mathrm{B}=1 \mathrm{~T}$ led to $16 \%$ power loss in one slowing down time, and to $28 \%$ power loss in two slowing down times. Losses with no pitch angle scattering are zero, as expected.

ORBITMN has been benchmarked for the calculation of the axisymmetric losses for QAS3_53 against the TRANSP code, which only simulates tokamak plasmas. Here both codes simulated axisymmetric losses assuming the same q profile, $1 \mathrm{~T}$ magnetic field, Zeff $=1.8,40 \mathrm{keV}$ beam ions using the high edge density beam deposition profile, only co-parallel injection and no charge exchange losses. Both also declared an ion lost when it crossed the last closed flux surface. The radial profiles of the collision rates and their magnitudes were similar. TRANSP finds $29 \%$ losses, evaluated for ions which have slowed down to near thermal energies $\sim 2-3 \mathrm{keV}$. This is in good agreement with the ORBIT prediction of $28 \%$ loss, for beam ions which after two slowing down times are at $2.72^{-2}$ of their initial energy or $\sim 5 \mathrm{keV}$. TRANSP simulations have shown that axisymmetric neutral beam ion losses improve with increased $\mathrm{B}$, proportional to $1 / \mathrm{B}^{2}$.

When charge exchange is included TRANSP finds 33\% energy loss. The charge exchange losses may be from a different class of particles than those lost due to stellarator ripple fields; in tokamaks these losses arise typically from passing ion orbits. Such ions would reside in the plasma long enough for charge exchange to occur. If this is also characteristic of stellarators, charge exchange and ripple losses will be additive. Further 
work is needed to clarify whether charge exchange and stellarator energetic ion losses are additive.

Including the full rippled field with both axisymmetric and nonaxisymmetric components, ORBITMN calculated $41 \%$ power loss in one slowing down time and $49 \%$ power loss in two slowing down times. With no pitch angle scattering, $17 \%$ energy losses are predicted, due to collisionless ripple loss. The time evolution of the collisionless loss $\left(v_{\perp}=0\right)$ is remarkable, characteristic of the stochastic field regions: $7 \%$ of the ions are "prompt" lost and another 10\% slowly leave the plasma over a characteristic time roughly one-third the energy slowing down time (Figure 10). Prompt single particle orbits are due to orbit wobble for ions deposited near the plasma edge, since the loss criterion is that the gyrocenter crosses the last closed flux surface. Delayed particle loss orbits exhibit motion characteristic of passing ion stochastic loss ${ }^{27-29}$. The delayed loss is only observed if $v_{\varepsilon} \neq 0$.

When pitch angle scattering is included, about $10 \%$ of the losses are "prompt". These are due to the rippled magnetic field near the plasma edge. The beam ion deposition interestingly does not lead to "prompt" losses in the axisymmetric field. Table IX shows beam ion loss results for 1) typical PBX-M values of density and temperature but Zeff lowered to 1.8, 2) parameters corresponding to the ISS95 transport scaling $^{30}$ and 3) a scan of $\langle\mathrm{Ne}>$ and $<\mathrm{Te}>$ to vary collisionality. Changing the density and temperature at the same Zeff is seen to vary the loss rate by less than $25 \%$, improving losses with reduced $\left\langle v_{\perp} / \nu_{\varepsilon}\right\rangle$ and for reduced $\tau_{\mathrm{e}}$.

For increased toroidal field $(\mathrm{B}=1.4 \mathrm{~T})$, the power losses remain at $39 \%$ in one slowing down time and $48 \%$ in two slowing down times, virtually no improvement, as 
was also found with the low beam ion edge deposition in the previous section. To examine the effect of launching the beam lines at different toroidal angle, considering the three dimensional shape of the stellarator, losses at $\phi=0$ (crescent cross section) were $41 \%$ in one slowing down time and at $\phi=\pi$ (oblate cross section) were $37 \%$. Beam loss can likely be improved by injecting at angles with reduced ripple losses as prompt losses within the first 25 toroidal transits were entirely suppressed at $\phi=\pi$, but rose to $12 \%$ at $\phi=0$, from $7 \%$ with random $\phi$.

A study was also made of the region of highest relative beam ion loss by truncating the beam deposition profile at several values of toroidal flux. It was found that beam ion losses rose linearly with minor radius outside the half radius, underlining the importance of reduced ripple fields.

\section{Section IV.C Measures of Stellarator Ripple}

Isaev et al. have developed a configuration optimization method to improve neoclassical transport using the pseudosymmetric concept ${ }^{31}$. A new measure of the level of symmetry breaking, or rippleness is denoted by the function W, called "Water". W is the total area of the ripple for half of one poloidal turn of the field line at the half radius. This is expected to better correlate with particle loss than simple measures of field line ripple, for example, $\mathrm{B}_{\mathrm{mn}}^{\max } / \mathrm{B}_{00}$, with which there is almost no correlation (Fig. 11). However, Fig. 12 shows that there is only modest correlation of co injected beam ion loss with W for the QAS stellarators. In particular QAS2_40 has high losses and small W. Large stellarator rippleness is in one case well correlated with high beam loss (QAS4_40). As discussed in the last section, the evaluation of relative neutral beam ion loss with 
radius showed that for QAS3_53 (1T), for example, coinjected parallel beam ion loss is high outside $0.5 \mathrm{r} / \mathrm{a}$. Since $\mathrm{W}$ is evaluated only at the half radius, it would be worthwhile to consider other measures of $\mathrm{W}$ such as 1) average $\mathrm{W}$ over all flux surfaces, 2) average over all flux surfaces outside the half radius or 3 ) over all flux surfaces, restricting $\theta$ to be near $\pm \pi / 2$ to reduce the loss of trapped ions.

From simulations of thermal ion diffusion, (Tables II and III) it is clear that the low thermal ion loss rate of QAS2_20 appears to be determined by the parameter $\mathrm{a}_{0,0}$, although it has nearly the same W as QAS3_53, three times that of QAS3_34. The width of the plasma appears to cancel the effect of high Bmax for QAS2_20. We expect large $\rho / a$ to be correlated with higher losses and QAS2_40 has much reduced $\mathrm{a}_{0,0}$ compared to QAS2_20 (19 vs 25). We conclude that field ripple should be reduced outside $0.5 \mathrm{r} / \mathrm{a}$ and that a larger midplane radius will improve neutral beam ion confinement in similar QAS designs. Finally, the stochastic loss of passing ions (Sec. IVB) may be controlled through development of a criterion for reduced stochastic loss domains.

\section{SIMULATIONS OF ALPHA PARTICLE TRANSPORT IN A QAS REACTOR}

Retention of fast ions will be essential for utilizing alpha particle and other fast ion heating in fusion reactors. In tokamaks, toroidal field ripple can drive high levels of fast particle stochastic ripple loss ${ }^{32}$, particularly in regions of high plasma q and q'. High ripple loss rates in reversed shear for $1 \mathrm{MeV}$ tritons were found in measurements of triton burnup $^{33}$ on Japan Atomic Energy Research Institute Tokamak-60 Upgrade (JT-60U) ${ }^{34}$ and in predictive simulations for the International Thermonuclear Experimental Reactor 
$(\text { ITER })^{35}$ of $3.5 \mathrm{MeV}$ alpha particles ${ }^{11,36,37}$. Similarly, high values of magnetic field ripple in a QAS reactor may cause unacceptable losses of fast ions.

To answer this question regarding the confinement and loss of alpha particles in a QAS reactor we chose the QAS2_20 configuration, which exhibited the best particle transport as well as ballooning stability at $B=1.4 \mathrm{~T}$. It was scaled to reactor size: $\mathrm{R}_{\text {maj }}=$ $453 \mathrm{~cm}, \mathrm{~B}=7.9 \mathrm{~T},\langle\mathrm{a}\rangle=220 \mathrm{~cm}$, with plasma characterized by $\left\langle\mathrm{N}_{\mathrm{e}}\right\rangle=2.3 \times 10^{14} / \mathrm{cm}^{3}, \mathrm{~N}_{\mathrm{eo}}$ $=4.7 \times 10^{14} / \mathrm{cm}^{3}, \mathrm{~T}_{\mathrm{e}}=25 \mathrm{keV}, \mathrm{Z}_{\mathrm{eff}}=2$. The collision rates were based on central plasma conditions and were constant with radius. The rates were given by $v_{\mathrm{pa}} / v_{\varepsilon}=0.07$, $v_{\perp}=1.03 / \mathrm{sec}, \quad v_{\varepsilon}=7.6 / \mathrm{sec}, \tau_{\varepsilon}=0.13 \mathrm{sec}$. The alpha profile was $\mathrm{N}_{\alpha}=\left(1-(\mathrm{r} / \mathrm{a})^{2}\right)^{9}$, similar to that used in TFTR and ITER simulations ${ }^{9-12}$.

Excellent confinement was found with no pitch angle scattering: $0.8 \%+/-0.4 \%$ alpha particles lost in $\tau_{\varepsilon}$. But simulations which included pitch angle scattering, led to 8 (12.5)\% alpha energy (particle) loss in one alpha particle slowing down time. Simulations of alpha loss in a comparable tokamak reactor, without TF ripple showed no alpha loss arising from pitch angle scattering over one slowing down time. These results with and without pitch angle scattering show that, as for the neutral beam ion loss simulations, the QAS alpha losses appear to be due primarily to scattering into ripple loss orbits, not due to low edge poloidal flux (trapped ions being 30\%). The combined effects of pitch angle scattering and ripple transport in the presence of many magnetic ripple wells increases alpha losses to levels higher than has been acceptable in ITER ( $\sim 5 \%$ alpha power loss corresponding to the lowest tolerable wall heat load $\left.{ }^{11}\right)$. At higher fields the alpha energy (particle) loss in one slowing down time is reduced to 1 (2)\%, if B is 
increased to $16 \mathrm{~T}$, unfortunately higher than is possible with contemporary magnetic coil technology.

For a tokamak, we would expect passing ion losses to be proportional to $1 /$ poloidal flux $=1 / \mathrm{BR}^{2}$. Tokamak trapped ion loss would to increase as the ratio $\delta / \delta_{\mathrm{s}}$ increases, where $\delta$ is a measure of the average field line ripple

$$
\delta=\left(\mathrm{B}_{\mathrm{MAX}}-\mathrm{B}_{\mathrm{MIN}}\right) /\left(\mathrm{B}_{\mathrm{MAX}}+\mathrm{B}_{\mathrm{MIN}}\right)
$$

and $\delta_{\mathrm{s}}$ is a stochastic threshold similar to the well known Goldston, White and Boozer (GWB) stochastic threshold ${ }^{28}$

$$
\delta_{\mathrm{GWB}}=(\varepsilon / \mathrm{N} \pi \mathrm{q})^{3 / 2}\left(1 / \rho q^{\prime}\right) .
$$

$\mathrm{N}$ is the number of toroidal field coils, $\rho$ is the ion gyro radius, $\varepsilon$ is the inverse aspect ratio $\mathrm{a} / \mathrm{R}$ and q' the plasma shear, $\mathrm{q}^{\prime}=\mathrm{r} \mathrm{dq} / \mathrm{dr}$. Because the threshold $\sim 1 / \rho \mathrm{q}^{\prime} \sim \mathrm{BR}$. We would expect trapped ion loss to increase faster than 1/BR. Increasing ion energy is similar to reduced magnetic field in increasing the threshold for stochastic loss. The loss will not drop smoothly with magnetic field since this is a threshold phenomenon but will rather drop off in steps reflecting the stochastic topology.

Figure 13 shows the simulation results for alpha loss from the QAS2_20 reactor as a function of $B$ and R. Neither particle nor energy losses follow the $1 / \mathrm{BR}^{2}$ behavior expected from edge poloidal flux considerations. Alpha particle loss in this QAS is also not controlled by edge poloidal flux - multiple stochastic regions cause a parametric dependence more like $1 / \mathrm{B}^{2} R$ for particle loss and $1 / \mathrm{B}^{2} \mathrm{R}^{2}$ for energy loss. The residual losses at very high field result from the presence of the stellarator stochastic fields and helical ripple wells. 
Poor alpha confinement was characteristic of early stellarator designs where any trapped alpha $\left(\mathrm{v}_{/ /}=0\right)$ was collisionlessly lost in a time less than an energy slowing down time $\sim 0.1$ second. New symmetric stellarator designs are configured to solve this historic problem by maintaining vanishing bootstrap current $\left(\mathrm{W} 7-\mathrm{X}^{38}\right.$, under construction in Germany) and with quasi-helical symmetry (HSX ${ }^{39}$, under construction in Wisconsin). These stellarators are predicted to confine collisionless alpha particles very well ${ }^{40}$. ORBITMN alpha loss simulations were carried out for the QAS configuration which had the most promising particle transport properties along with ballooning stability. Yet relatively high levels of alpha loss are predicted. Improved alpha particle confinement and collection of lost alphas at the plasma edge through a cleverly planned divertor are likely to be important for stellarator reactors as it has been for tokamak reactor designs. Calculations of collisional alpha transport for W7-X and HSX scaled to reactor size are also needed.

\section{CONCLUSION}

A variety of toroidal magnetic configurations of size $R=145 \mathrm{~cm}$ and field strength $\mathrm{B}=1 \mathrm{~T}$ have been studied with the new, Hamiltonian coordinate, Monte Carlo, particle following code ORBITMN, to evaluate the confinement of thermal and energetic plasma ions for compact QAS. The code was successfully benchmarked aginst GTC for QAS thermal ion transport and against TRANSP for the axisymmetric component of neutral beam ion transport. Several ballooning stable equilibrium magnetic geometries with $\beta<$ 7\% were investigated. The configurations considered varied widely in their particle transport properties. Comparisons were made to equivalent tokamak configurations 
(without TF ripple) showing that tokamak transport is better by a factor of at least 2 , when measured by diffusion coefficients for thermal ions and by beam ion loss fractions for neutral beam heating ions.

From simulations of $40 \mathrm{KeV}$ neutral beam ions, the calculated loss fractions were found to strongly favor parallel, co-injection into low Zeff plasmas. Neutral beam ion loss fractions were high, being several times the losses of comparable tokamaks. A parallel injected beam in the promising QAS3_53, scaled to $\mathrm{R}=145 \mathrm{~cm}, \mathrm{~B}=1 \mathrm{~T}$, lost a high percentage of power: $28 \%$ of the beam energy and $51 \%$ of the beam ions in one slowing down time. With a more realistic beam deposition profile having a higher edge deposition the power losses increased to $41 \%$ in one slowing down time.

In general, QAS energetic particle loss fractions and thermal ion diffusion coefficients showed a similar functional dependence on $\Psi_{\text {edge }}$. Both types of simulation studies are needed for quantitative comparisons. Pitch angle scattering drives fast passing ions into trapped ion orbits which are rapidly lost in regions of stochastic fields and helical ripple wells. This makes the survival of even parallel injected beam ions strongly dependent on the number and size of the stochastic field regions and ripple wells, not primarily on the edge poloidal flux. In addition, collisionless beam ion simulations exhibited stochastic passing ion loss. Nevertheless, thermal ion transport can be minimized with reduced ripple fields and, less effectively than for tokamaks, by higher edge poloidal flux. Neutral beam ion fuelling and heating losses will also be minimized with lower field ripple and will be lowest for experiments with small values of $v_{\perp} / \nu_{\varepsilon}$, and of $\tau_{\varepsilon}$. Good neutral beam ion confinement at moderate ripple in QAS2_20 suggests that 
larger minor radius designs with reduced $\rho / a$ will be important to include in configuration optimization. A strategy to minimize stochastic beam ion loss is needed.

Simulations of alpha loss from the QAS configuration with the best particle confinement, scaled to reactor size, showed relatively high alpha loss, compared to ITER Interim Design simulations. Reduction of alpha loss through lower ripple fields should be a high priority in the search for QAS reactor configurations. Collisional alpha transport simulations for other new stellarator designs, such as those with helical axisymmety, with vanishing bootstrap current and those with constrained omnigeneity, should also be of high priority in reactor design.

\section{ACKNOWLEDGEMENTS}

We are grateful to R. J. Goldston, R. J. Hawryluk and J. Hosea for their interest and encouragement. We are glad to thank L-P. Ku, and D. Monticello for providing the magnetic configurations. It is also a pleasure to acknowledge S. P. Hirshman for use of the VMEC equilibrium code and W. A. Cooper for the TERPSICHORE ballooning stability code. These codes made it possible for the NCSX Team to identify good candidate magnetic configurations with quasi-axisymmetric symmetry. We also thank R. Budny and D. McCune for providing the TRANSP analysis for PBX-M. M. Suewattana held a DOE National Undergraduate Fellowship at PPPL during the summer of 1998. This research was supported by U. S. Department of Energy Contract No. DEAC02-76-CH03073. 


\section{REFERENCES}

a) Present address: Physics Department, Lehigh University, Bethlehem, PA 18015.

[1] J. Nuehrenberg, W. Lotz and S. Gori, in Theory of Fusion Plasmas, E. Sindoni, F. Troyon and J. Vaclavik, eds., SIF, Bologna, 1994.

[2] P. R. Garabedian, Phys. Plasmas 3, 2483 (1996).

[3] H. Renner, W7AS Team, NBI Group, ICF Group and ECRH Group, Plasma Phys. Controlled Fusion 31, 1579 (1980).

[4] A. H. Boozer, Plasma Physics and Controlled Fusion 37, A103 (1995). A. H. Boozer, Phys. Plas. 5, 1647 (1998).

[5] A. H. Boozer, Plasma Physics Reports 23, 483 (1997). A. Reiman, L. P. Ku, D. Monticello, et al. Proceedings of the Seventeenth International Conference on Plasma Physics and Controlled Nuclear Fusion Research (Yokahama, Japan) October, 1998. (International Atomic Energy Agency, Vienna, Austria) to be published.

[6] R. E. Bell, N. Asakur, S. Bernabei, et al., Phys. Fluids B2, 1271 (1990). B. LeBlanc, S. Batha, R. Bell, et al., Phys. Plas. 2, 741 (1995).

[7] H. Eubank, R. Goldston, J. Hosea, et al, Phys. Rev. Lett. 43, 270 (1979).

[8] G. Y. Fu, L. P. Ku, N. Pomphry, et al., Proceedings of the Seventeenth International Conference on Plasma Physics and Controlled Nuclear Fusion Research (Yokahama, Japan) October, 1998. (International Atomic Energy Agency, Vienna, Austria) to be published.

[9] M. H. Redi, M. C. Zarnstorff, R. B. White, R. V. Budny, A. C. Janos, D. K. Owens, J. F. Schivell, S. D. Scott and S. J. Zweben, Nuclear Fusion 35, 1191 (1995). [10] M. H. Redi, R. V. Budny, D. S. Darrow, H. H. Duong, R. K. Fisher, A. C. 
Janos, J. M. McChesney, D. C. McCune, S. S. Medley, M. P. Petrov, J. F. Schivell, S. D. Scott, R. B. White, M. C. Zarnstorff and S. J. Zweben, Nuclear Fusion 35, 1509 (1995).

[11] M. H. Redi, R. V. Budny, C. O. Miller, R. B. White, Phys. Plas. 3, 3037 (1996).

[12] M. H. Redi, R. B. White, S. H. Batha, F. M. Levinton, D. C. McCune, Physics of Plasmas, 4, 4001 (1997).

[13] S. J. Zweben, D. S. Darrow, S. H. Batha, et al, Nuclear Fusion, 38, 739 (1998).

[14] K. Tani, T. Takizuka, M. Azumi, H. Kishimoto, Nucl. Fusion 23, 657 (1983).

[15] R. B. White, R. J. Goldston, M. H. Redi, R. V. Budny, Phys. Plas., 3, 3043 (1996).

[16] R. B. White and M. S. Chance, Phys. Fluids 27, 2455 (1984) .

[17] S. P. Hirshman and J. C. Whitson, Phys. Fluids 26, 2553 (1983)

[18] F. Najmadbadi, the ARIES Team: C. G. Bathe, M. C. Billane, et al, Fusion Engineering and Design 38 (1997) 1-218.

[19] W. A. Cooper, et al, Nucl. Fusion 29, 617 (1989).

[20] H. Mynick, Plasma Physics Reports 23, 547 (1997).

[21] R. B. White, Theory of Tokamak Plasmas, Princeton University Press, Princeton, NJ, (1989) p. 74-84.

[22] J. Wesson, Tokamaks, Oxford University Press, Oxford, UK, (1987) p. 92-93.

[23] R. V. Budny, D. C. McCune, M. H. Redi, J. Schivell, R. M. Wieland, Phys. Plas. 3, 4583 (1996).

[24] D. L. Book, Publ. 177-4405, NRL Plasma Formulary, Washington, D.C. (1990) $64 \mathrm{pp}$. 
[25] R. J. Hawryluk, D. Mueller, J. Hosea, et al, Fusion Tech. 21, 1324 (1992).

[26] R. J. Goldston, H. H. Towner, J. Plasma Physics 26, 283 (1981).

[27] H. E. Mynick, Phys. Plas. 5, 1471 (1993).

[28] H. E. Mynick, Phys. Plas. 5, 2460 (1993).

[29] S. V. Konovalov, S. V. Putvinskii, Sov. J. Plasma Phys. 14, 461 (1988).

[30] U. Stroth, M . Murakami, R. A. Dory, H. Yamada, S. Okamura, F. Sano, T. Obiki, Nuclear Fusion 36, 1063 (1996).

[31] M. Yu. Isaev, L. P. Ku, M. I. Mikhailov, D. A. Monticello, H. E. Mynick, A. H. Reiman, A. A. Subbotin, "Optimization of NCSX towards Pseudosymmetry", in preparation.

[32] R. J. Goldston, R. B. White and A. H. Boozer, Phys. Rev. Lett. 47, 647 (1981) .

[33] K. Tobita, Nuclear Fusion 35, 1585 (1995).

[34] H. Ninomiya and the JT60 Team, Phys. Fluids B 4, 2070 (1992).

[35] P.-H. Rebut, V. Chuyanov, M. Huguet, R. Parker and Y. Shimomura, in Plasma Physics and Controlled Nuclear Fusion Research 1994, Proceedings of the 15th International Conference, Seville, 1994 (International Atomic Energy Agency, Vienna, 1996), Vol. 2, p. 451.

[36] S. V. Konovalov, ITER Physics Design report, International Atomic Energy Agency, Vienna, 1996.

[37] M. H. Redi, R. B. White, R. J. Goldston, in Plasma Physics and Controlled Nuclear Fusion Research 1996 (Proc. 23rd E. P. S. Conf. Kiev, Ukraine 1996), European Physical Society, Petit-Lancy. 
[38] G. Grieger, et al., Plasma Physics and Controled Nuclear Fusion Research, (Proc.

13th Int. Conf., Washington, 1990) Vol. 3, 525 IAEA, Vienna (1991).

[39] D. T. Anderson, Proc. J. Plasma Fusion Res. SERIES, Vol 1 (1998).

[40] W. Lotz, P. Merkel, J. Nuhrenberg, E. Strumberger, Plasma Physics and Controlled Fusion 34, 1037 (1992). 
TABLE I. Toroidal magnetic configurations

Number of Field Periods $\quad$ External Transform (\%) Beta(\%)

Quasi-axisymmetric Stellarators

$\begin{array}{llll}\text { QAS2_20 } & 2 & 20 & 6.7 \\ \text { QAS2_40 } & 2 & 40 & 5.8 \\ \text { QAS3_34 } & 3 & 34 & 3.9 \\ \text { QAS3_53 } & 3 & 53 & 3.9 \\ \text { QAS4_40 } & 4 & 40 & 7.2\end{array}$

Equivalent and Analytic Tokamaks

$\begin{array}{llll}\text { TOKQAS2_20 } & 1 & 0 & 6.7 \\ \text { TOKQAS2_20q } & 1 & 0 & 6.6 \\ \text { TOKQAS3_53 } & 1 & 0 & 4.6 \\ \text { TOK0 } & 1 & 0 & 6.2\end{array}$


TABLE II. Thermal ion loss rates for quasi-axisymmetric stellarators and equivalent tokamaks

400 transit times, 1000 particles

40 transit times, 4000 particles

\begin{tabular}{lcccc}
\hline & Fractional loss & D & Fractional loss & D \\
& $\%$ & $\mathrm{~cm}^{2} / \mathrm{sec}$ & $\%$ & $\mathrm{~cm}^{2} / \mathrm{sec}$ \\
& & & & \\
\hline QAS2_20 & 3 & 7690 & 0.03 & 8570 \\
QAS2_40 & 23 & -- & 1.3 & 15000 \\
QAS3_34 & 32 & -- & 0.5 & 8330 \\
QAS3_53 & 40 & -- & 0.6 & 5600 \\
QAS3_53 (1T) & 65 & -- & 17 & -- \\
QAS4_40 & 11 & -- & 4 & 12000 \\
TOKQAS2_20 & 2 & 8000 & & -- \\
TOKQAS2_20q & 0 & 4769 & 0 & -- \\
TOKQAS3_53 (1T) & -- & -- & 0 & - \\
TOK0 & 65 & -- & 22 & \\
\hline & & & & \\
\hline
\end{tabular}


TABLE III. Parameters of magnetic geometry and diffusion coefficients

$\begin{array}{cccccc}\langle\mathrm{a}\rangle_{\text {stell }} & \langle\mathrm{a}\rangle_{0,0} & \mathrm{~W} & \mathrm{q}_{\mathrm{a}} & \Psi_{\text {edge }} & \mathrm{D} \\ (\mathrm{cm}) & (\mathrm{cm}) & & & (\mathrm{W}) & \left(\mathrm{cm}^{2} / \mathrm{sec}\right)\end{array}$

$\begin{array}{llllllr}\text { QAS2_20 } & 51 & 25 & 0.0020 & 2.7 & 0.66 & 8570 \\ \text { QAS2_40 } & 49 & 22 & 0.0013 & 2.4 & 0.65 & 15,000 \\ \text { QAS3_34 } & 37 & 19 & 0.0022 & 2.5 & 0.22 & 8330 \\ \text { QAS3_53 } & 37 & 22 & 0.0098 & 2.2 & 0.22 & 5600 \\ \text { QAS3_53 (1T) } & 37 & 22 & -- & 2.2 & 0.16 & -- \\ \text { QAS4_40 } & 49 & 24 & 0.052 & 2.9 & 0.43 & 12000 \\ & & & & & & 8000 \\ \text { TOKQAS2_20 } & 56 & 33 & 0 & 7.0 & 0.31 & 4769 \\ \text { TOKQAS2_20q } & 56 & 35 & 0 & 2.8 & 0.72 & 3600 \\ \text { TOKQAS3_53(1T) } & 38 & 21 & 0 & 2.2 & .019 & --\end{array}$


TABLE IV. Ratio of collision rate to bounce frequency, $v *=v / \omega_{b}$

Thermal ions Neutral Beam Alpha Particles

\begin{tabular}{lcc}
\hline QAS2_20 & 0.03 & 0.001 \\
QAS2_40 & 0.03 & 0.001 \\
QAS4_40 & 0.07 & 0.002 \\
QAS3_34 & 0.12 & 0.002 \\
QAS3_53 & 0.40 & 0.002 \\
TOKQAS2_20 & 0.05 & \\
TOKQAS2_20q & 0.05 & 0.0001 \\
TOK0 & 0.05 & 0.0001 \\
\hline & & \\
\hline
\end{tabular}


TABLE V. Neutral beam ion loss during $\tau_{\varepsilon}$ from QAS2_20, for $v_{\perp}(0)=35 / \mathrm{sec}, v_{\varepsilon}=37 / \mathrm{sec}$

\begin{tabular}{|c|c|c|c|c|}
\hline $\mathbf{R}_{\mathrm{inj}}$ & Co/Counter & Parallel/Perpendicular & Energy Loss & Particle Loss \\
\hline$(\mathrm{cm})$ & & & $(\%)$ & $(\%)$ \\
\hline
\end{tabular}

With no pitch angle scattering, $B z=1.4 \mathrm{~T}$

$\begin{array}{rllcc}130 & \text { co } & \text { parallel } & 0.4 & 0.4 \\ 34 & \text { co } & \text { perpendicular } & 1.3 & 1.6 \\ -34 & \text { counter } & \text { perpendicular } & 36 & 41 \\ -130 & \text { counter } & \text { parallel } & 1.5 & 1.5\end{array}$

With pitch angle scattering, $\mathrm{Bz}=1.4 \mathrm{~T}$

\begin{tabular}{|c|c|c|c|}
\hline 130 & co & parallel & 7 \\
\hline 34 & co & perpendicular & 53 \\
\hline-34 & counter & perpendicular & 72 \\
\hline-130 & counter & parallel & 26 \\
\hline
\end{tabular}

With pitch angle scattering, $\mathrm{Bz}=2.8 \mathrm{~T}$

130

co parallel

3

6 
TABLE VI. Neutral beam ion loss during $\tau_{\varepsilon}$ from QAS2_20, for $v_{\perp}(0)=17 / \mathrm{sec}, v_{\varepsilon}=17 / \mathrm{sec}$

$\mathbf{R}_{\mathrm{inj}}$

$(\mathrm{cm})$
Co/Counter Parallel/Perpendicular Energy Loss

$(\%)$
Particle Loss

$(\%)$

With no pitch angle scattering, $B z=1.4 \mathrm{~T}$

$\begin{array}{rllcc}130 & \text { co } & \text { parallel } & 0.4 & 0.4 \\ 34 & \text { co } & \text { perpendicular } & 4 & 4 \\ -34 & \text { counter } & \text { perpendicular } & 53 & 57 \\ -130 & \text { counter } & \text { parallel } & 0.4 & 0.4\end{array}$

With pitch angle scattering, $\mathrm{Bz}=1.4 \mathrm{~T}$

$\begin{array}{rllcr}130 & \text { co } & \text { parallel } & 21 & 39 \\ 34 & \text { co } & \text { perpendicular } & 72 & 88 \\ -34 & \text { counter } & \text { perpendicular } & 88 & 96 \\ -130 & \text { counter } & \text { parallel } & 47 & 70\end{array}$

With pitch angle scattering, $\mathrm{Bz}=\mathbf{2 . 8} \mathrm{T}$

co

parallel

6

11 
TABLE VII. Parallel coinjected neutral beam ion loss during $\tau_{\varepsilon}$ for toroidal confinement designs of PBX-M size for $v_{\varepsilon}=37 / \mathrm{sec},\left\langle v_{\perp} / v_{\varepsilon}\right\rangle=0.67$

Low edge deposition profile

Energy loss Particle loss

(\%)
$(\%)$
High edge deposition profile Energy loss Particle loss

(\%)

(\%)

\section{QAS stellarators}

$\begin{array}{lcc}\text { QAS2_20 } & 7 & 12 \\ \text { QAS2_40 } & 27 & 49 \\ \text { QAS3_34 } & 14 & 27 \\ \text { QAS3_53 } & 26 & 48 \\ \text { QAS4_40 } & 25 & 45 \\ \text { QAS3_53(1T) } & 28 & 51\end{array}$

41

65

\section{Tokamaks}

$\begin{array}{lcl}\text { TOKQAS2_20 } & 10 & 18 \\ \text { TOKQAS2_20q } & 0 & \\ \text { TOKQAS3_53(1T) } & 16 & 32\end{array}$


TABLE VIII. Neutral beam ion loss during $\tau_{\varepsilon}$ for QAS3_53 (1T)

$\left(<v_{\perp} / v_{\varepsilon}>=0.67, v_{\varepsilon}=37 / \mathrm{sec}\right)$

\begin{tabular}{lcccc}
$\mathbf{R}_{\text {inj }}$ & Co/Counter & Parallel/Perpendicular & Energy Loss & Particle Loss \\
$(\mathrm{cm})$ & & & $(\%)$ & $(\%)$ \\
\hline 130 & co & parallel & 28 & 51 \\
34 & co & perpendicular & 76 & 91 \\
-34 & counter & perpendicular & 100 & 100 \\
-130 & counter & parallel & 79 & 91
\end{tabular}


TABLE IX. Collisionality dependence of neutral beam ion loss from QAS3_53(1T) with high edge deposition

\begin{tabular}{|c|c|c|c|c|c|c|}
\hline \multirow[t]{2}{*}{$\langle\mathrm{Ne}\rangle$} & \multirow[t]{2}{*}{$\langle\mathrm{Te}\rangle$} & \multirow[t]{2}{*}{$\left\langle v_{\perp}\right\rangle$} & \multirow[t]{2}{*}{$v_{\mathrm{e}}$} & \multirow[t]{2}{*}{$\left\langle v_{\perp} / v_{\mathrm{e}}\right\rangle$} & \multicolumn{2}{|c|}{ E loss } \\
\hline & & & & & $\tau_{\mathrm{e}}$ & $2 \tau_{\mathrm{e}}$ \\
\hline $4 \mathrm{e} 13 / \mathrm{cm}^{3 \mathrm{a}}$ & $1.3 \mathrm{keV}$ & $24 / \mathrm{s}$ & $35 / \mathrm{s}$ & 0.67 & $41 \%$ & $49 \%$ \\
\hline $4 \mathrm{e} 13 / \mathrm{cm}^{3} \mathrm{~b}$ & $1.3 \mathrm{keV}$ & $0 / \mathrm{s}$ & $35 / \mathrm{s}$ & 0 & $17 \%$ & \\
\hline $6 . \mathrm{e} 13 / \mathrm{cm}^{3 \mathrm{c}}$ & $1.3 \mathrm{keV}$ & $34 / \mathrm{s}$ & $50 / \mathrm{s}$ & 0.67 & $41 \%$ & $50 \%$ \\
\hline 3 e 19 & 2.65 & 26 & 18 & 1.0 & 48 & \\
\hline 5 e 19 & 2.42 & 44 & 23 & 1.3 & 53 & \\
\hline 5 e 19 & 1.9 & 44 & 26 & 1.2 & 51 & \\
\hline 7 e 19 & 1.75 & 62 & 40 & 1.1 & 47 & \\
\hline 9 e 19 & 1.55 & 79 & 56 & 1.0 & 46 & \\
\hline a Plasma parameter & PBX-M & Zeff $=$ & & & & \\
\hline b Plasma parameter & PBX-M & , Zeff $=$ & & & & \\
\hline${ }^{\mathrm{c}}$ ISS95 transport sc & ing. & & & & & \\
\hline
\end{tabular}




\section{List of figure captions}

Figure 1.
a) Magnetic flux surfaces at $\phi=0$ of the QAS2_20 configuration.
b) Magnetic flux surfaces at $\phi=0$ of the QAS2_40 configuration.
c) Magnetic flux surfaces at $\phi=0$ of the QAS3_34 configuration.
d) Magnetic flux surfaces at $\phi=0$ of the QAS4_40 configuration.
e) Magnetic flux surfaces at $\phi=0$ of the TOKQAS2_20 and TOKQAS2_20q configurations.

f) Magnetic flux surfaces at $\phi=0$ of the TOK0 configuration.

Figure 2.
a) Q versus toroidal flux for the QAS2_20 and TOKQAS2_20q configurations.
b) Q versus toroidal flux for the QAS2_40 configuration.
c) Q versus toroidal flux for the QAS_34 configuration.
d) Q versus toroidal flux for the QAS4_40 configuration.
e) Q versus toroidal flux for for the TOKQAS2_20 configuration.
f) Q versus toroidal flux for for the TOK0 configuration.

Figure 3.

Initial thermal ion density profile versus edge normalized toroidal flux $\Phi / \Phi($ a).

Figure 4.
a) Final ion density profiles after 400 toroidal transits versus normalized toroidal flux $\Phi / \Phi($ a) for the QAS2_20 configuration.
b) Final ion density profiles after 400 toroidal transits versus normalized toroidal flux $\Phi / \Phi($ a) for the QAS2_40 configuration. 
c) Final ion density profiles after 400 toroidal transits versus normalized toroidal flux $\Phi / \Phi($ a) for the QAS3_34 configuration.

d) Final ion density profiles after 400 toroidal transits versus normalized toroidal flux $\Phi / \Phi($ a) for the QAS4_40 configuration.

e) Final ion density profiles after 400 toroidal transits versus normalized toroidal flux $\Phi / \Phi($ a) for the TOKQAS2_20 configuration.

f) Final ion density profiles after 400 toroidal transits versus normalized toroidal flux $\Phi / \Phi($ a) for the TOK0 configuration.

Figure 5.

a) Thermal ion loss fraction time evolution versus number of toroidal transits for the QAS2_20 configuration.

b) Thermal ion loss fraction time evolution versus number of toroidal transits for the QAS2_40 configuration.

c) Thermal ion loss fraction time evolution versus number of toroidal transits for the QAS3_34 configuration.

d) Thermal ion loss fraction time evolution versus number of toroidal transits for the QAS4_40 configuration.

e) Thermal ion loss fraction time evolution versus number of toroidal transits for the TOKQAS2_20 configuration.

f) Thermal ion loss fraction time evolution versus number of toroidal transits for the TOK0 configuration.

Figure 6.

a) Diffusion coefficient function $\mathrm{f}(\mathrm{t})$ evolution for the QAS2_20 configuration. 
b) Diffusion coefficient function $\mathrm{f}(\mathrm{t})$ evolution for the QAS2_40 configuration.

c) Diffusion coefficient function $\mathrm{f}(\mathrm{t})$ evolution for the QAS3_34 configuration.

d) Diffusion coefficient function $\mathrm{f}(\mathrm{t})$ evolution for the QAS4_40 configuration.

e) Diffusion coefficient function $\mathrm{f}(\mathrm{t})$ evolution for the TOKQAS2_20

configuration.

f) Diffusion coefficient function $\mathrm{f}(\mathrm{t})$ evolution for the TOK0 configuration.

Figure 7.

Diffusion coefficients versus edge poloidal flux for QAS and equivalent tokamak configurations.

Figure 8.

Initial beam ion deposition profiles with low and high edge deposition.

Figure 9.

Parallel, co-injected neutral beam ion loss fractions versus edge poloidal flux for QAS and equivalent tokamak configurations.

Figure 10.

Time evolution of the fraction of collisionless particle loss for parallel co-injected beam ions from QAS3_53(1T) over one energy slowing down time.

Figure 11

Dependence of neutral beam ion losses for QAS stellarators at $1.4 \mathrm{~T}, \mathrm{R}=145 \mathrm{~cm}$ on $\mathrm{B}_{\mathrm{mn}}^{\max } / \mathrm{B}_{00}$, a relatively simple measure of stellarator symmetry breaking.

Figure 12

Dependence of neutral beam ion losses for QAS stellarators at $1.4 \mathrm{~T}, \mathrm{R}=145 \mathrm{~cm}$ on $\mathrm{W}$, a measure of stellarator symmetry breaking. 
Figure 13.

Alpha particle loss fractions for the reactor size scaled QAS2_20 configuration dependence on magnetic field strength and major radius. 

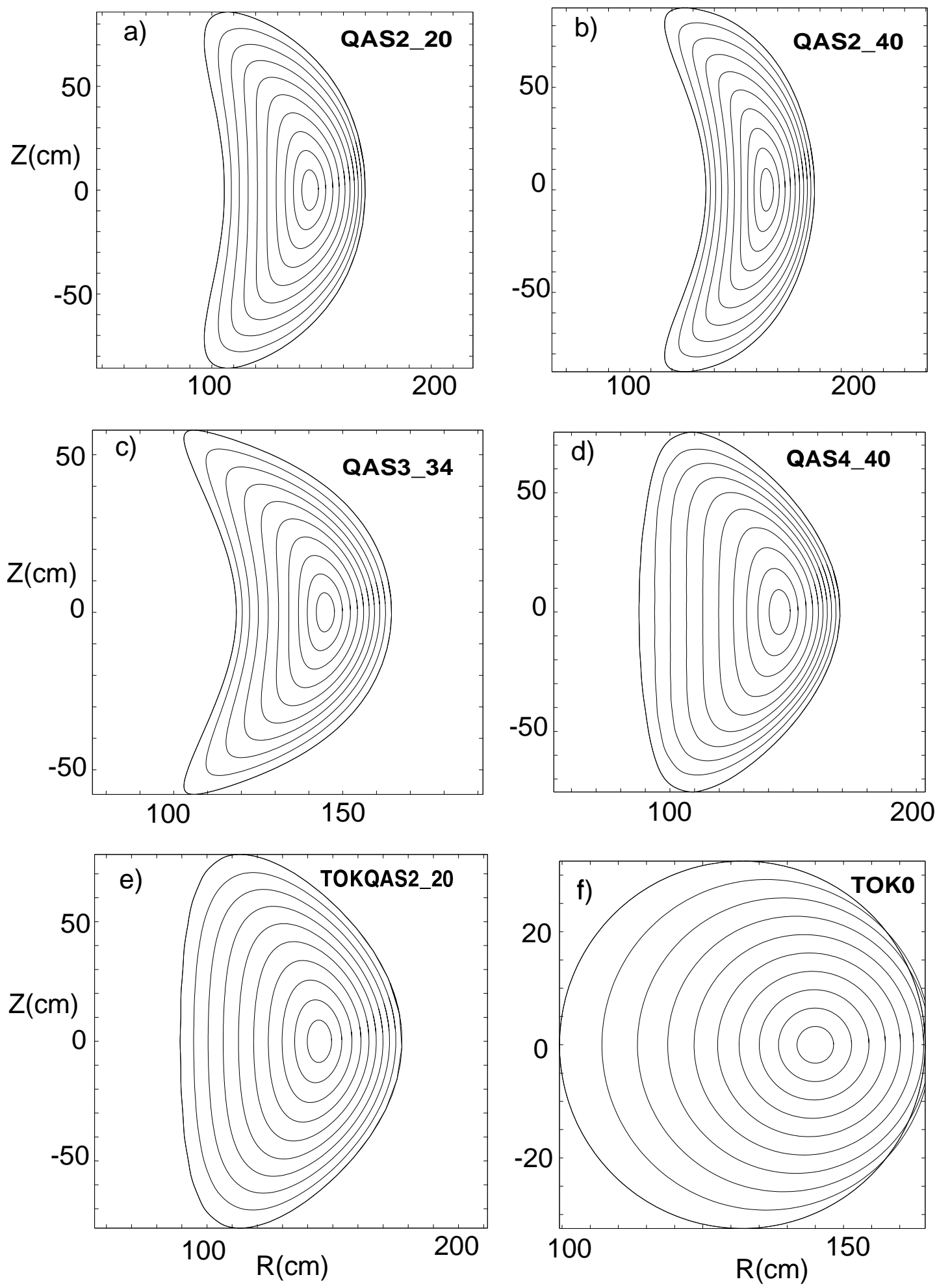

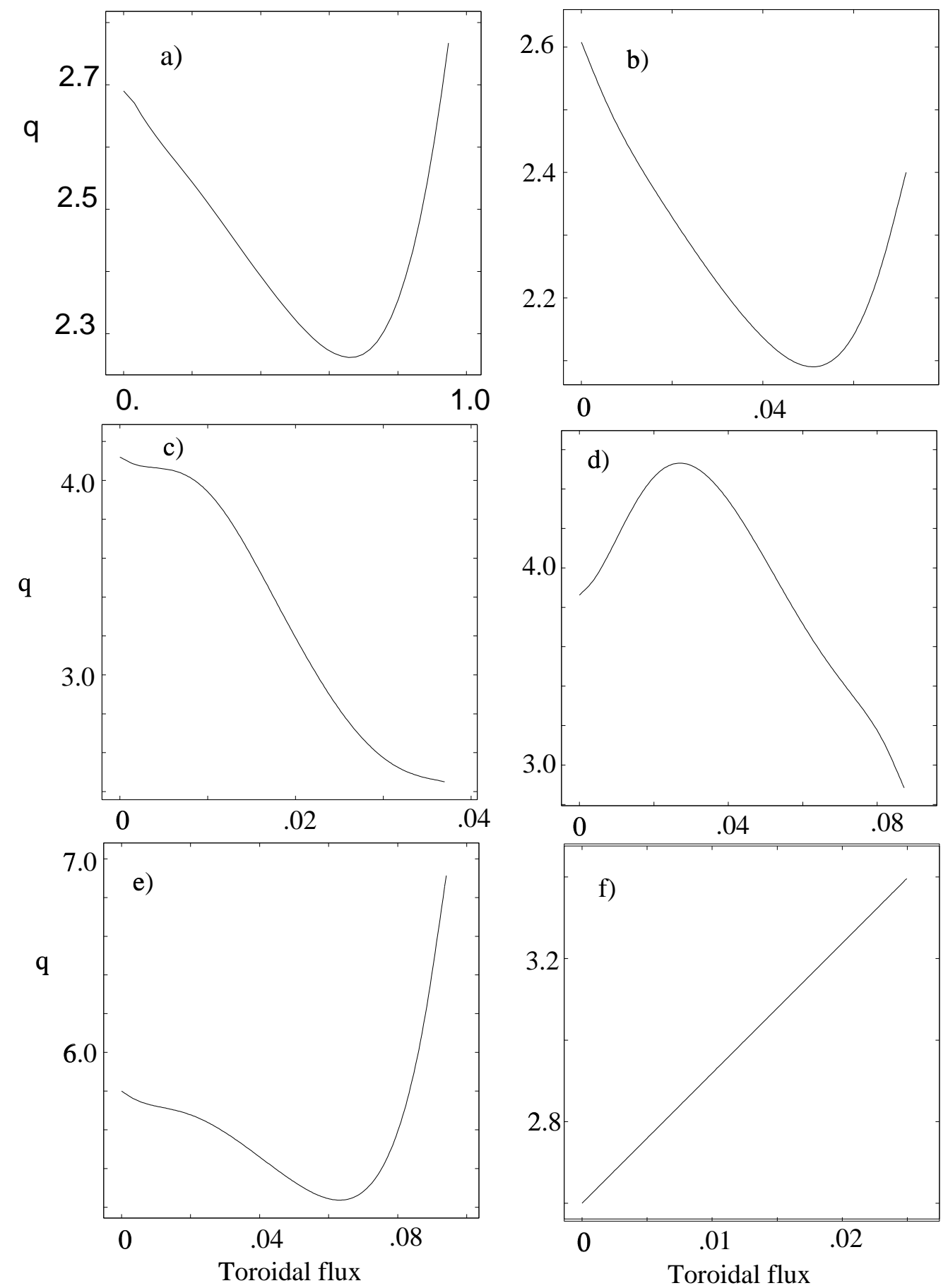


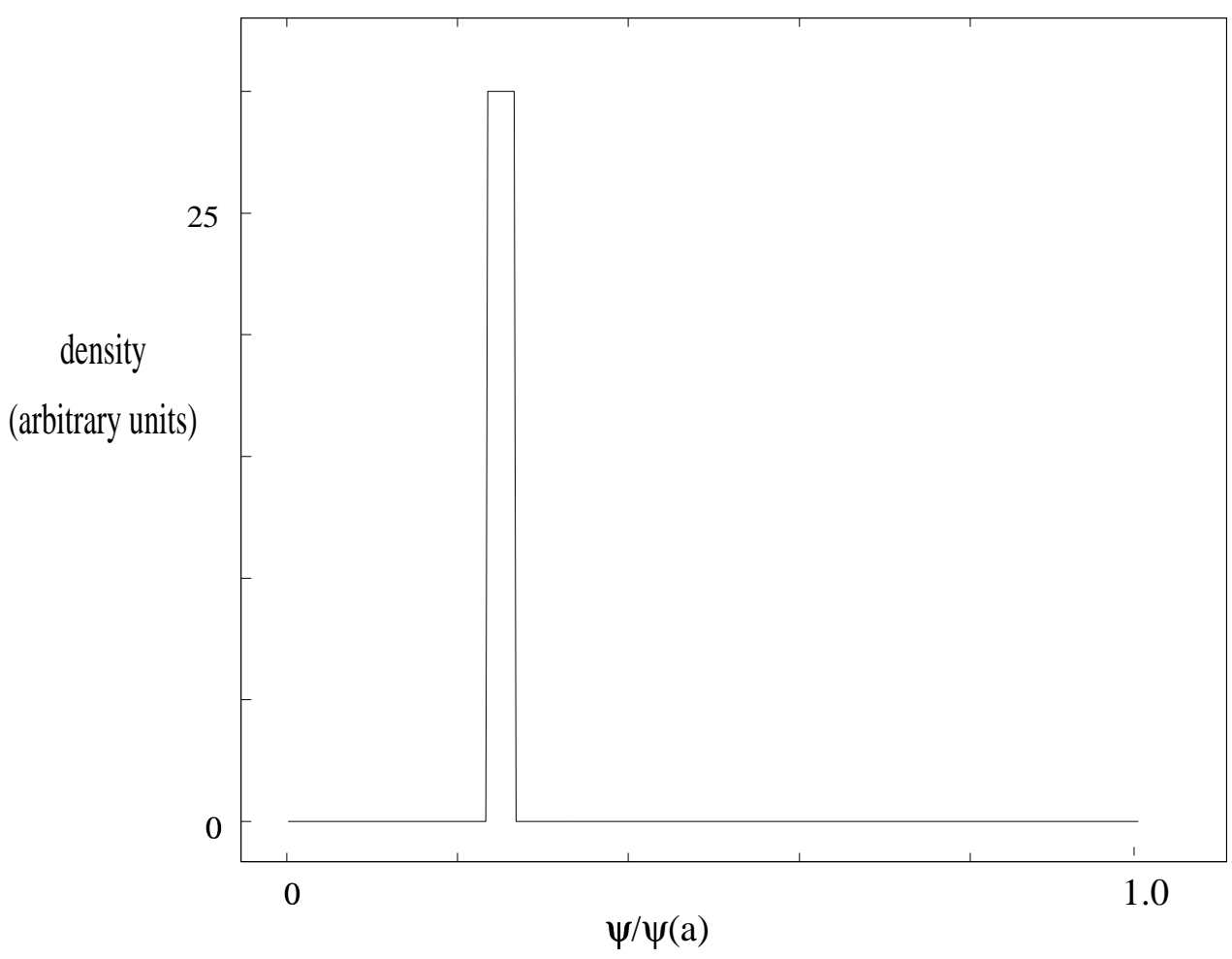



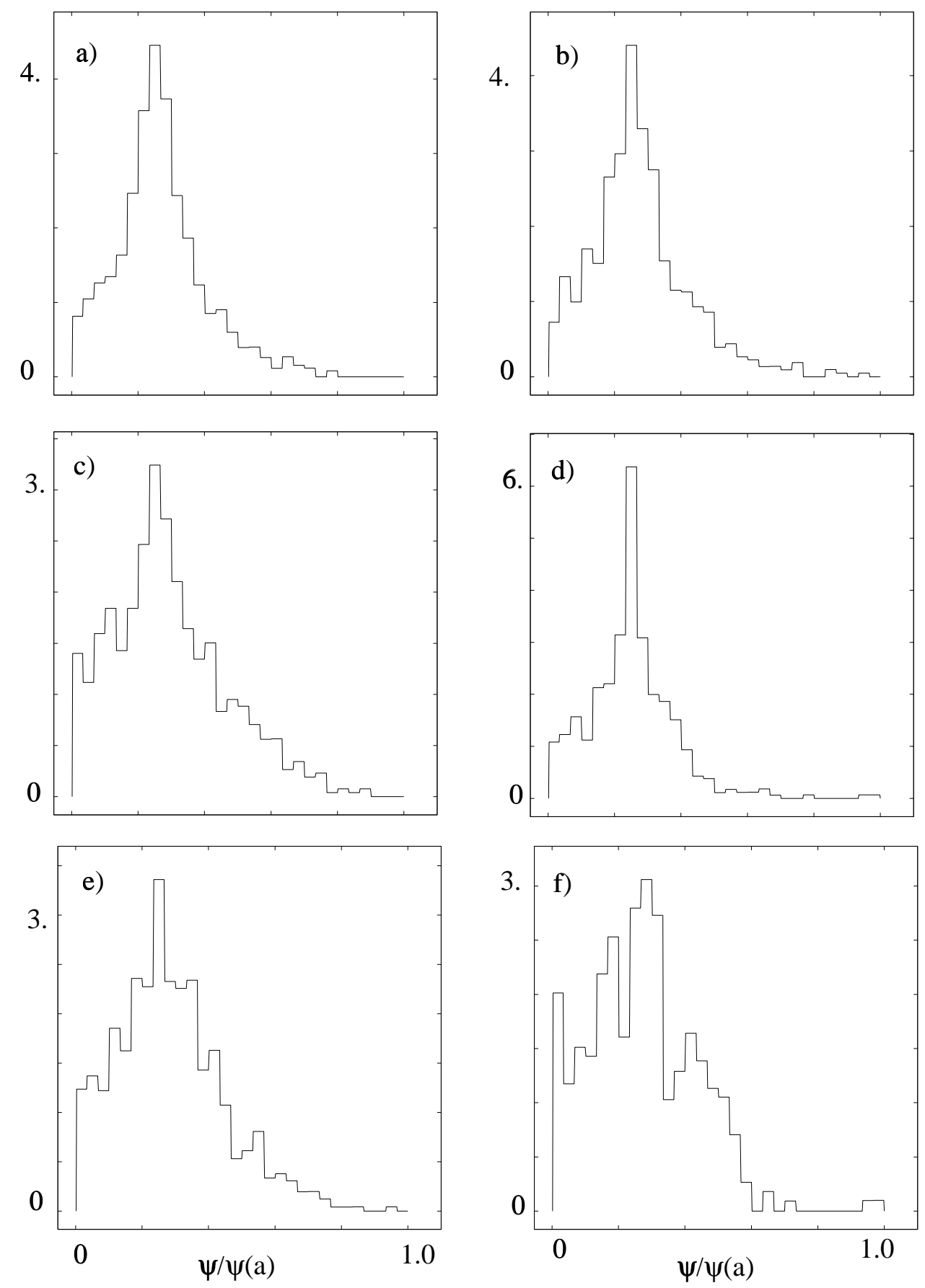

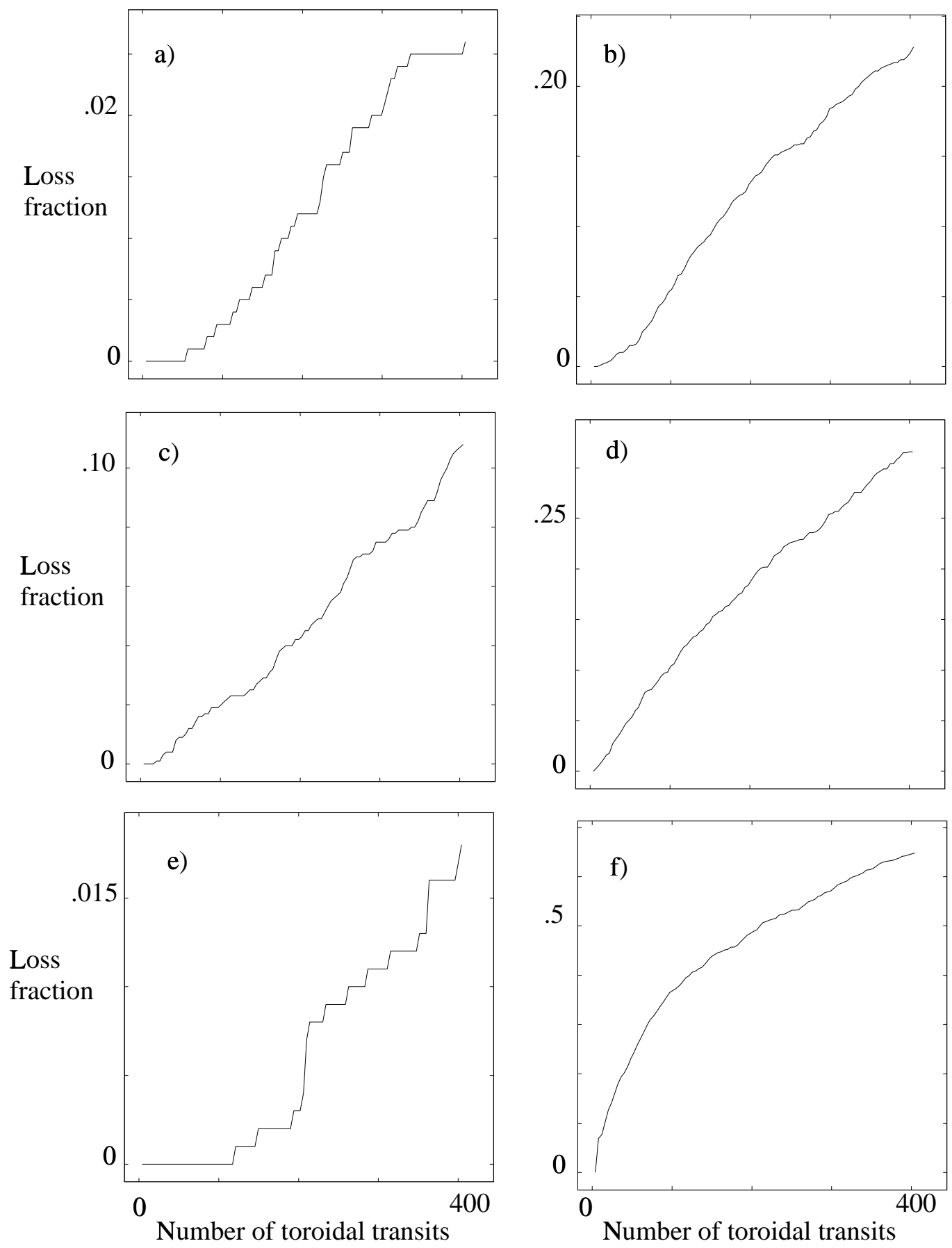

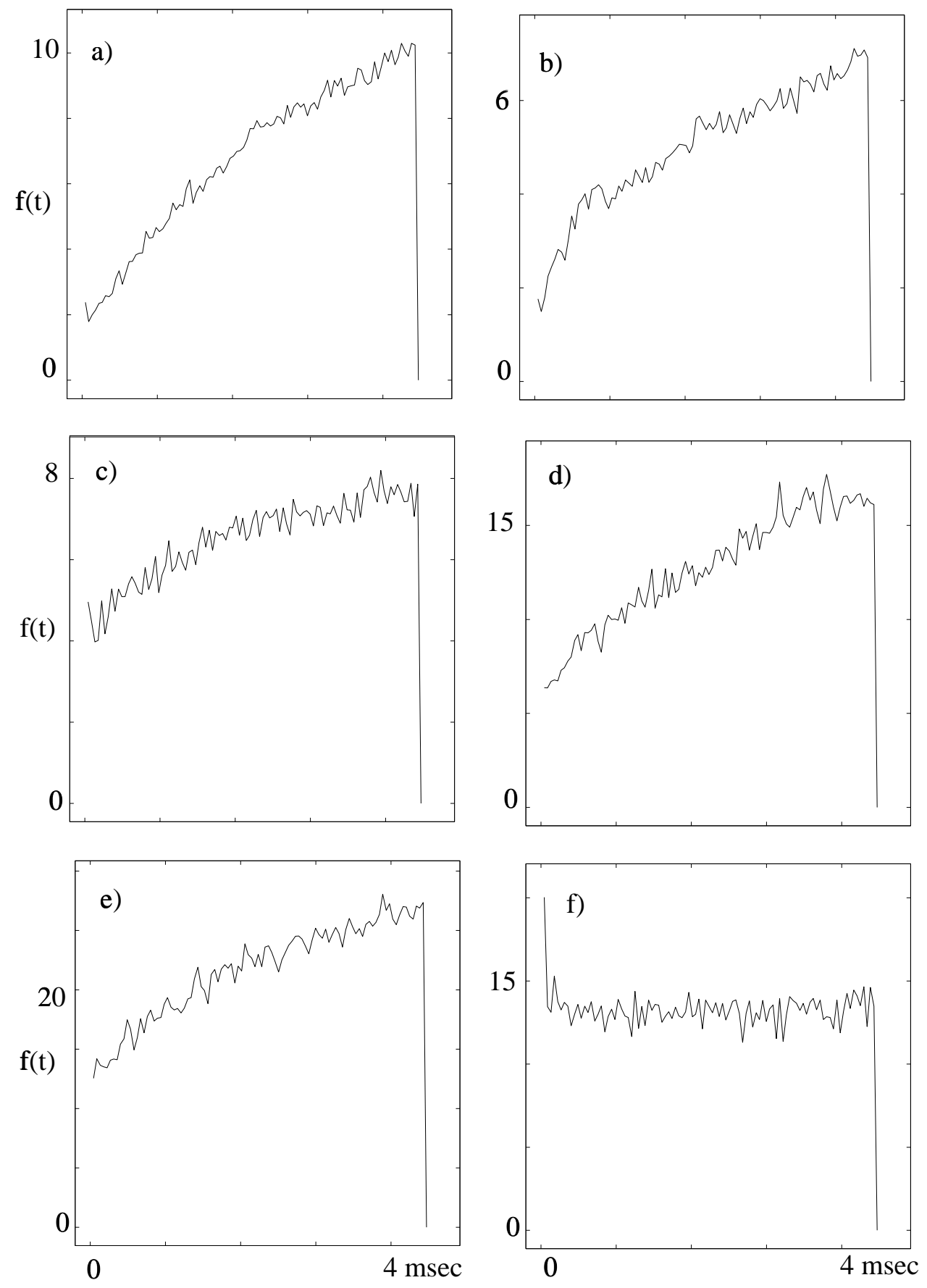


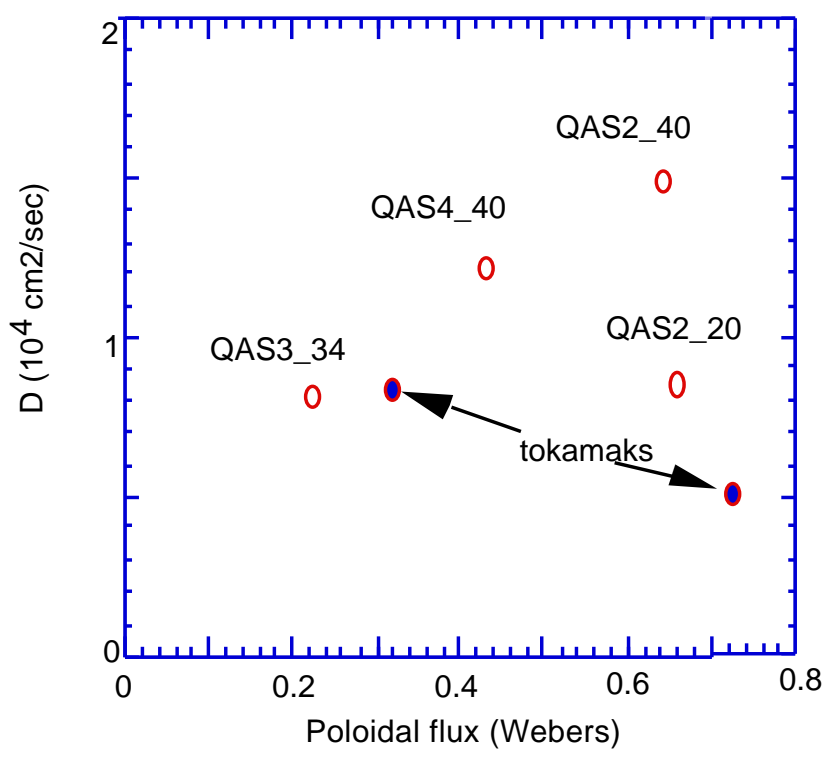

Figure 7, Redi 


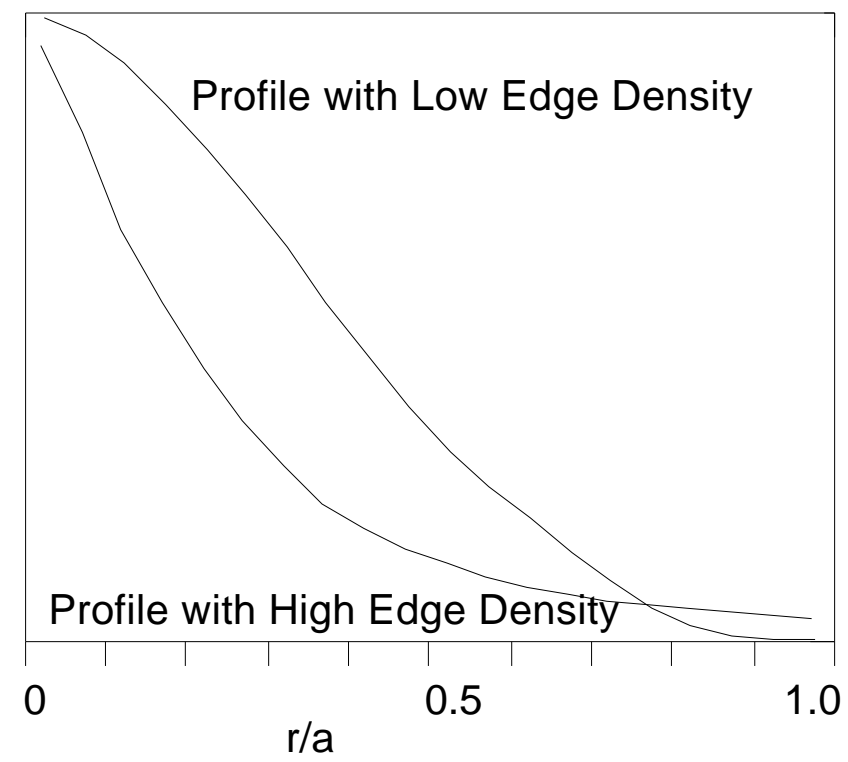

Figure 8, Redi 


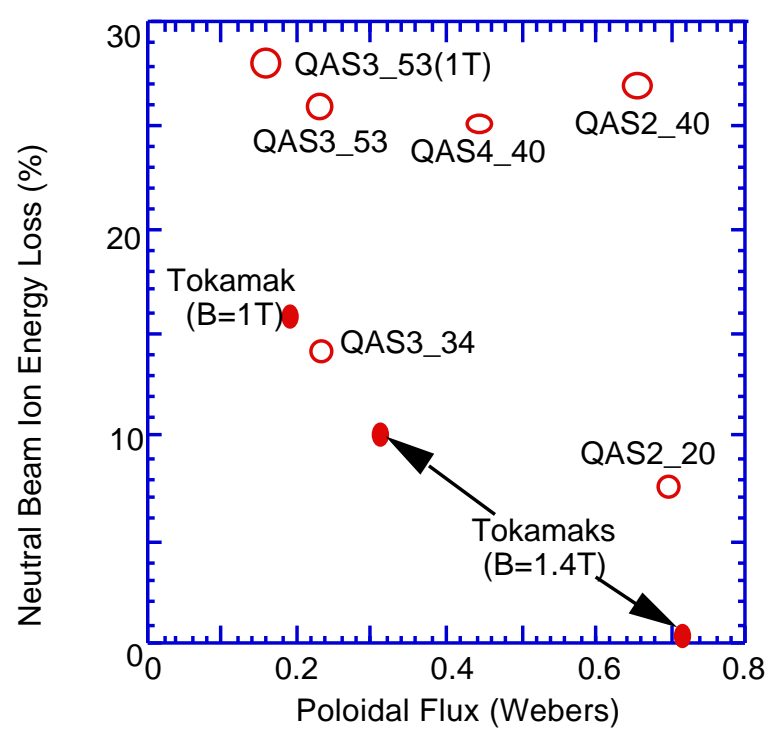

Figure 9, Redi 


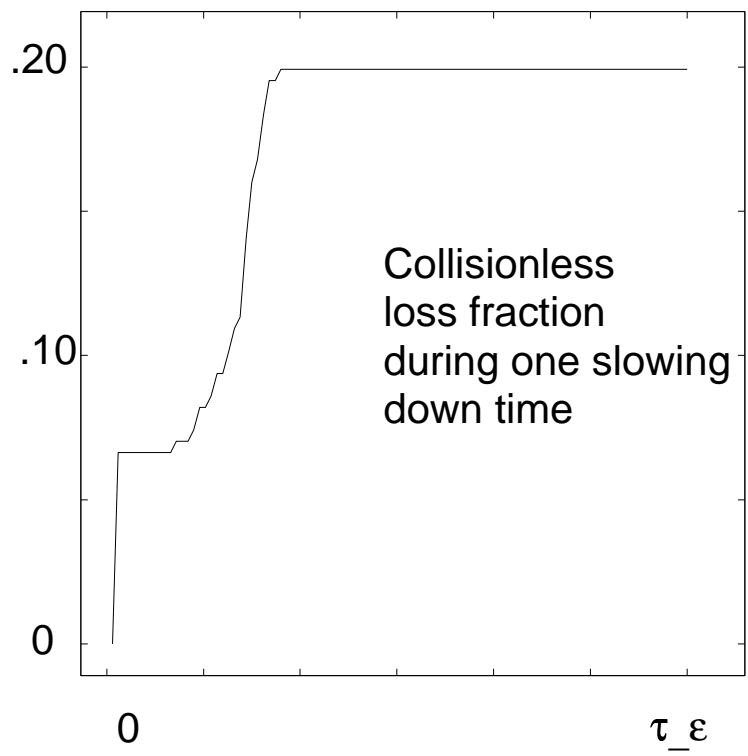

Figure 10, Redi 


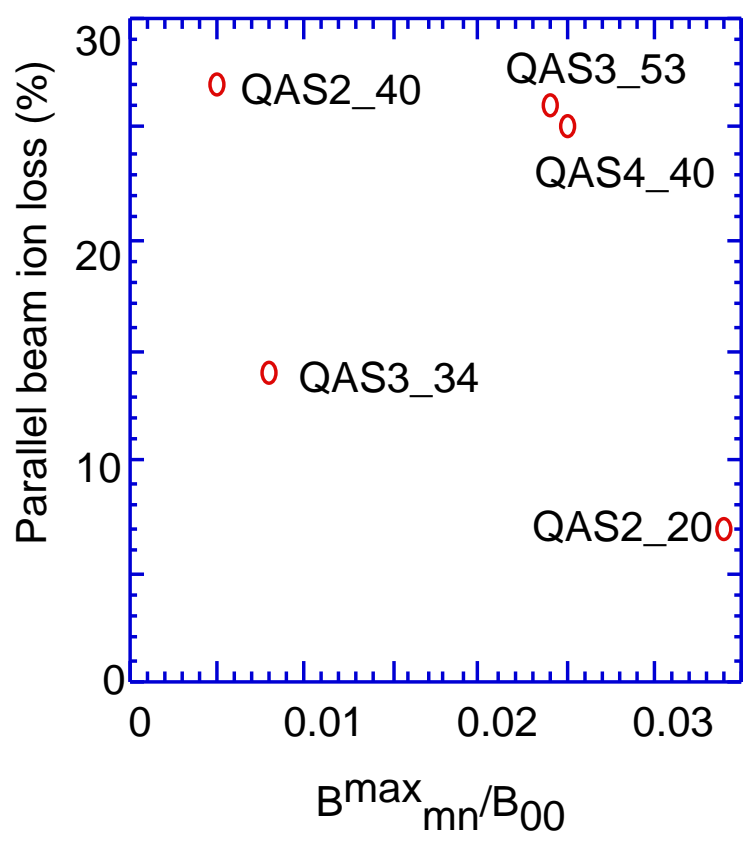

Figure 11, Redi 


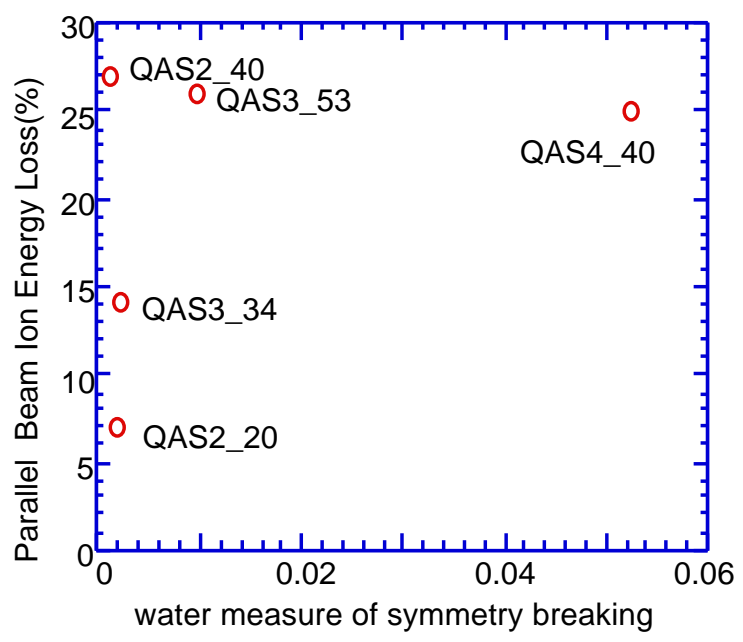

Fig 12, Redi 


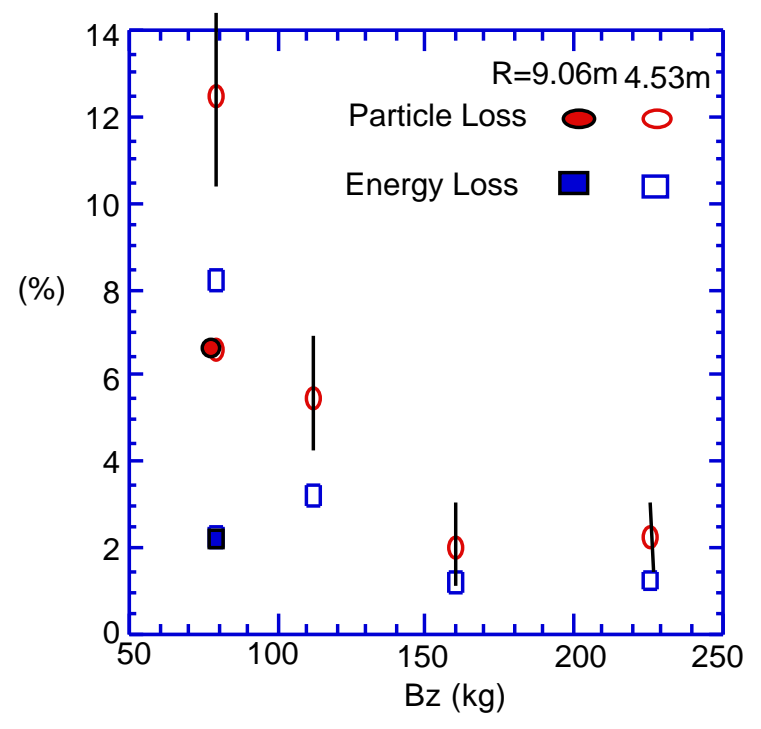

Figure 13 